\title{
A Precise Measurement of the Tau Polarization and its Forward-Backward Asymmetry at LEP
}

\author{
The OPAL Collaboration
}

\begin{abstract}
A measurement of the $\tau$ lepton polarization and its forward-backward asymmetry at the $\mathrm{Z}^{0}$ resonance using the OPAL detector is described. The measurement is based on analyses of $\tau \rightarrow \rho \nu_{\tau}, \tau \rightarrow \pi(\mathrm{K}) \nu_{\tau}, \tau \rightarrow \mathrm{e} \bar{\nu}_{e} \nu_{\tau}, \tau \rightarrow \mu \bar{\nu}_{\mu} \nu_{\tau}$, and $\tau \rightarrow \mathrm{a}_{1} \nu_{\tau}$ decays from a sample of $89075 \mathrm{e}^{+} \mathrm{e}^{-} \rightarrow \tau^{+} \tau^{-}$candidates corresponding to an integrated luminosity of $117 \mathrm{pb}^{-1}$. Assuming that the $\tau$ lepton decays according to $\mathrm{V}-\mathrm{A}$ theory, we measure the average $\tau$ polarization at $\sqrt{s}=\mathrm{M}_{\mathrm{Z}}$ to be $\left\langle P_{\tau}\right\rangle=(-13.0 \pm 0.9 \pm 0.9) \%$ and the $\tau$ polarization forward-backward asymmetry to be $\mathrm{A}_{\mathrm{pol}}^{\mathrm{FB}}=(-9.4 \pm 1.0 \pm 0.4) \%$, where the first error is statistical and the second systematic. These results are consistent with the hypothesis of lepton universality and, when combined, can be expressed as a measurement of $\sin ^{2} \theta_{\text {eff }}^{\text {lept }}=$ $0.2334 \pm 0.0012$ within the context of the Standard Model.
\end{abstract}

to be submitted to Z. Phys. 


\section{The OPAL Collaboration}

G. Alexander ${ }^{23}$, J. Allison ${ }^{16}$, N. Altekamp ${ }^{5}$, K. Ametewee ${ }^{25}$, K.J. Anderson ${ }^{9}$, S. Anderson ${ }^{12}$, S. Arcelli ${ }^{2}$, S. Asai ${ }^{24}$, D. Axen ${ }^{29}$, G. Azuelos ${ }^{18, a}$, A.H. Ball ${ }^{17}$, E. Barberio ${ }^{26}$, R.J. Barlow ${ }^{16}$, R. Bartoldus ${ }^{3}$, J.R. Batley ${ }^{5}$, G. Beaudoin ${ }^{18}$, J. Bechtluft ${ }^{14}$, C. Beeston ${ }^{16}$, T. Behnke ${ }^{8}$, A.N. Bell ${ }^{1}$, K.W.Bell ${ }^{20}$, G. Bella ${ }^{23}$, S. Bentvelsen ${ }^{8}$, P. Berlich ${ }^{10}$, S. Bethke ${ }^{14}$, O. Biebel ${ }^{14}$, V. Blobel ${ }^{8}$, I.J. Bloodworth ${ }^{1}$, J.E. Bloomer ${ }^{1}$, P. Bock ${ }^{11}$, H.M. Bosch ${ }^{11}$, M. Boutemeur ${ }^{18}$, B.T. Bouwens ${ }^{12}$, S. Braibant ${ }^{12}$, R.M. Brown ${ }^{20}$, H.J. Burckhart ${ }^{8}$, C. Burgard ${ }^{27}$, R. Bürgin ${ }^{10}$, P. Capiluppi ${ }^{2}$, R.K. Carnegie ${ }^{6}$, A.A. Carter ${ }^{13}$, J.R. Carter ${ }^{5}$, C.Y. Chang ${ }^{17}$, C. Charlesworth ${ }^{6}$, D.G. Charlton ${ }^{1, b}$, D. Chrisman ${ }^{4}$, S.L. Chu ${ }^{4}$, P.E.L. Clarke ${ }^{15}$, I. Cohen ${ }^{23}$, J.E. Conboy ${ }^{15}$, O.C. Cooke ${ }^{16}$, M. Cuffiani ${ }^{2}$, S. Dado ${ }^{22}$, C. Dallapiccola ${ }^{17}$, G.M. Dallavalle ${ }^{2}$, S. De Jong ${ }^{12}$, L.A. del Pozo ${ }^{8}$, K. Desch ${ }^{3}$, M.S. Dixit ${ }^{7}$, E. do Couto e Silva ${ }^{12}$, M. Doucet ${ }^{18}$, E. Duchovni ${ }^{26}$, G. Duckeck ${ }^{8}$, I.P. Duerdoth ${ }^{16}$, J.E.G. Edwards ${ }^{16}$, P.G. Estabrooks ${ }^{6}$, H.G. Evans ${ }^{9}$, M. Evans ${ }^{13}$,

F.Fabbri ${ }^{2}$, P. Fath ${ }^{11}$, F. Fiedler ${ }^{12}$, M. Fierro ${ }^{2}$, H.M. Fischer ${ }^{3}$, R. Folman ${ }^{26}$, D.G. Fong ${ }^{17}$, M. Foucher ${ }^{17}$, H. Fukui ${ }^{24}$, A. Fürtjes ${ }^{8}$, P. Gagnon ${ }^{7}$, A. Gaidot ${ }^{21}$, J.W. Gary ${ }^{4}$, J. Gascon ${ }^{18}$, S.M. Gascon-Shotkin ${ }^{17}$, N.I. Geddes ${ }^{20}$, C. Geich-Gimbel ${ }^{3}$, F.X. Gentit ${ }^{21}$, T. Geralis ${ }^{20}$, G. Giacomelli ${ }^{2}$, P. Giacomelli ${ }^{4}$, R. Giacomelli ${ }^{2}$, V. Gibson ${ }^{5}$, W.R. Gibson ${ }^{13}$, D.M. Gingrich ${ }^{30, a}$, J. Goldberg ${ }^{22}$, M.J. Goodrick ${ }^{5}$, W. Gorn ${ }^{4}$, C. Grandi ${ }^{2}$, E. Gross ${ }^{26}$, M. Gruwée ${ }^{8}$, C. Hajdu ${ }^{32}$, G.G. Hanson ${ }^{12}$, M.Hansroul ${ }^{8}$, M. Hapke ${ }^{13}$, C.K.Hargrove ${ }^{7}$, P.A.Hart ${ }^{9}$, C. Hartmann ${ }^{3}$, M. Hauschild ${ }^{8}$, C.M. Hawkes ${ }^{5}$, R. Hawkings ${ }^{8}$, R.J. Hemingway ${ }^{6}$, G. Herten ${ }^{10}$, R.D. Heuer ${ }^{8}$, M.D. Hildreth ${ }^{8}$, J.C. Hill ${ }^{5}$, S.J. Hillier ${ }^{1}$, T. Hilse ${ }^{10}$, J. Hoare ${ }^{5}$, P.R. Hobson ${ }^{25}$, R.J. Homer ${ }^{1}$, A.K. Honma ${ }^{28, a}$, D. Horváth ${ }^{32, c}$, R. Howard ${ }^{29}$, R.E. Hughes-Jones ${ }^{16}$, D.E. Hutchcroft ${ }^{5}$, P. Igo-Kemenes ${ }^{11}$, D.C. Imrie ${ }^{25}$, M.R. Ingram ${ }^{16}$, A. Jawahery ${ }^{17}$, P.W. Jeffreys ${ }^{20}$, H. Jeremie ${ }^{18}$, M. Jimack ${ }^{1}$, A. Joly ${ }^{18}$, C.R. Jones ${ }^{5}$, G. Jones ${ }^{16}$, M. Jones ${ }^{6}$, R.W.L. Jones ${ }^{8}$, U. Jost ${ }^{11}$, P. Jovanovic ${ }^{1}$, T.R. Junk ${ }^{8}$, D. Karlen ${ }^{6}$, K. Kawagoe ${ }^{24}$, T. Kawamoto ${ }^{24}$, R.K. Keeler ${ }^{28}$, R.G.Kellogg ${ }^{17}$, B.W.Kennedy ${ }^{20}$, B.J. King ${ }^{8}$, J. Kirk ${ }^{29}$, S. Kluth ${ }^{8}$, T. Kobayashi ${ }^{24}$, M.Kobel ${ }^{10}$, D.S. Koetke ${ }^{6}$, T.P. Kokott ${ }^{3}$, S. Komamiya ${ }^{24}$, R. Kowalewski ${ }^{8}$, T. Kress ${ }^{11}$, P. Krieger ${ }^{6}$, J. von Krogh $^{11}$, P. Kyberd ${ }^{13}$, G.D. Lafferty ${ }^{16}$, H. Lafoux ${ }^{21}$, R. Lahmann ${ }^{17}$, W.P. Lai ${ }^{19}$, D. Lanske ${ }^{14}$, J. Lauber $^{15}$, S.R. Lautenschlager ${ }^{31}$, J.G. Layter ${ }^{4}$, D. Lazic ${ }^{22}$, A.M. Lee ${ }^{31}$, E. Lefebvre ${ }^{18}$, D. Lellouch ${ }^{26}$, J. Letts ${ }^{2}$, L. Levinson ${ }^{26}$, C. Lewis ${ }^{15}$, S.L. Lloyd ${ }^{13}$, F.K. Loebinger ${ }^{16}$, G.D. Long ${ }^{17}$, M.J. Losty ${ }^{7}$, J. Ludwig ${ }^{10}$, A. Luig ${ }^{10}$, A. Malik ${ }^{21}$, M. Mannelli ${ }^{8}$, S. Marcellini ${ }^{2}$, C. Markus ${ }^{3}$, A.J. Martin ${ }^{13}$, J.P. Martin ${ }^{18}$, G. Martinez ${ }^{17}$, T. Mashimo ${ }^{24}$, W. Matthews ${ }^{25}$, P. Mättig ${ }^{3}$, W.J. McDonald ${ }^{30}$, J. McKenna ${ }^{29}$, E.A. Mckigney ${ }^{15}$, T.J. McMahon ${ }^{1}$, A.I. McNab ${ }^{13}$,

R.A.McPherson ${ }^{8}$, F. Meijers ${ }^{8}$, S. Menke ${ }^{3}$, F.S. Merritt ${ }^{9}$, H. Mes ${ }^{7}$, J. Meyer ${ }^{27}$, A. Michelini ${ }^{2}$, G. Mikenberg ${ }^{26}$, D.J. Miller ${ }^{15}$, R. Mir $^{26}$, W.Mohr ${ }^{10}$, A. Montanari ${ }^{2}$, T. Mori ${ }^{24}$, M. Morii ${ }^{24}$, U. Müller ${ }^{3}$, H.A. Neal ${ }^{8}$, B. Nellen ${ }^{3}$, B. Nijjhar ${ }^{16}$, R. Nisius ${ }^{8}$, S.W. O'Neale ${ }^{1}$, F.G. Oakham ${ }^{7}$, F. Odorici ${ }^{2}$, H.O. Ogren ${ }^{12}$, T. Omori ${ }^{24}$, M.J. Oreglia ${ }^{9}$, S. Orito ${ }^{24}$, J. Pálinkás ${ }^{33, d}$, J.P.Pansart ${ }^{21}$, G.Pásztor ${ }^{32}$, J.R.Pater ${ }^{16}$, G.N.Patrick ${ }^{20}$, M.J. Pearce ${ }^{1}$, S.Petzold ${ }^{27}$, P. Pfeifenschneider ${ }^{14}$, J.E. Pilcher ${ }^{9}$, J. Pinfold ${ }^{30}$, D.E. Plane ${ }^{8}$, P. Poffenberger ${ }^{28}$, B. Poli ${ }^{2}$, A. Posthaus ${ }^{3}$, H. Przysiezniak ${ }^{30}$, D.L. Rees ${ }^{1}$, D. Rigby ${ }^{1}$, S.A. Robins ${ }^{13}$, N. Rodning ${ }^{30}$, J.M. Roney ${ }^{28}$, A. Rooke ${ }^{15}$, E. Ros ${ }^{8}$, A.M. Rossi ${ }^{2}$, M. Rosvick ${ }^{28}$, P. Routenburg $^{30}$, Y. Rozen ${ }^{8}$, K. Runge ${ }^{10}$, O. Runolfsson ${ }^{8}$, U.Ruppel ${ }^{14}$, D.R. Rust ${ }^{12}$, R. Rylko ${ }^{25}$, E.K.G.Sarkisyan ${ }^{23}$, M.Sasaki ${ }^{24}$,

C.Sbarra ${ }^{2}$, A.D.Schaile ${ }^{8, e}$, O.Schaile ${ }^{10}$, F.Scharf ${ }^{3}$, P.Scharff-Hansen ${ }^{8}$, P.Schenk ${ }^{4}$,

B. Schmitt ${ }^{3}$, S. Schmitt ${ }^{11}$, M. Schröder ${ }^{8}$, H.C. Schultz-Coulon ${ }^{10}$, M. Schulz ${ }^{8}$, P. Schütz ${ }^{3}$, W.G.Scott ${ }^{20}$, T.G.Shears ${ }^{16}$, B.C.Shen ${ }^{4}$, C.H.Shepherd-Themistocleous ${ }^{27}$, P. Sherwood ${ }^{15}$, G.P.Siroli ${ }^{2}$, A.Sittler ${ }^{27}$, A.Skillman ${ }^{15}$, A.Skuja ${ }^{17}$, A.M.Smith ${ }^{8}$, T.J.Smith ${ }^{28}$, G.A.Snow ${ }^{17}$, 
R. Sobie ${ }^{28}$, S. Söldner-Rembold ${ }^{10}$, R.W.Springer ${ }^{30}$, M.Sproston ${ }^{20}$, A.Stahl ${ }^{3}$, M.Starks ${ }^{12}$, M. Steiert ${ }^{11}$, K.Stephens ${ }^{16}$, J.Steuerer ${ }^{27}$, B. Stockhausen ${ }^{3}$, D. Strom ${ }^{19}$, F. Strumia ${ }^{8}$,

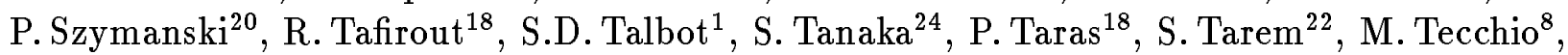
M. Thiergen ${ }^{10}$, M.A. Thomson ${ }^{8}$, E. von Törne ${ }^{3}$, S. Towers ${ }^{6}$, M. Tscheulin ${ }^{10}$, T. Tsukamoto ${ }^{24}$, E. Tsur ${ }^{23}$, A.S. Turcot ${ }^{9}$, M.F. Turner-Watson ${ }^{8}$, P. Utzat ${ }^{11}$, R. Van Kooten ${ }^{12}$, G. Vasseur ${ }^{21}$, M. Verzocchi ${ }^{10}$, P. Vikas ${ }^{18}$, M. Vincter ${ }^{28}$, E.H. Vokurka ${ }^{16}$, F. Wäckerle ${ }^{10}$, A. Wagner ${ }^{27}$,

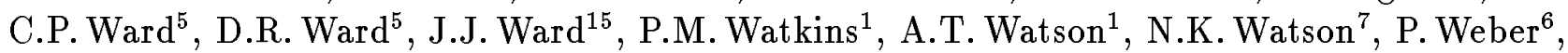
P.S. Wells ${ }^{8}$, N. Wermes ${ }^{3}$, J.S. White ${ }^{28}$, B. Wilkens ${ }^{10}$, G.W. Wilson ${ }^{27}$, J.A. Wilson ${ }^{1}$, T. Wlodek ${ }^{26}$, G. Wolf ${ }^{26}$, S. Wotton ${ }^{5}$, T.R. Wyatt ${ }^{16}$, S. Yamashita ${ }^{24}$, G. Yekutieli ${ }^{26}$, V. Zacek ${ }^{18}$,

${ }^{1}$ School of Physics and Space Research, University of Birmingham, Birmingham B15 2TT, UK 2Dipartimento di Fisica dell' Università di Bologna and INFN, I-40126 Bologna, Italy

${ }^{3}$ Physikalisches Institut, Universität Bonn, D-53115 Bonn, Germany

${ }^{4}$ Department of Physics, University of California, Riverside CA 92521, USA

${ }^{5}$ Cavendish Laboratory, Cambridge CB3 0HE, UK

${ }^{6}$ Ottawa-Carleton Institute for Physics, Department of Physics, Carleton University, Ottawa, Ontario K1S 5B6, Canada

${ }^{7}$ Centre for Research in Particle Physics, Carleton University, Ottawa, Ontario K1S 5B6, Canada

${ }^{8}$ CERN, European Organisation for Particle Physics, CH-1211 Geneva 23, Switzerland

${ }^{9}$ Enrico Fermi Institute and Department of Physics, University of Chicago, Chicago IL 60637, USA

${ }^{10}$ Fakultät für Physik, Albert Ludwigs Universität, D-79104 Freiburg, Germany

${ }^{11}$ Physikalisches Institut, Universität Heidelberg, D-69120 Heidelberg, Germany

${ }^{12}$ Indiana University, Department of Physics, Swain Hall West 117, Bloomington IN 47405, USA

${ }^{13}$ Queen Mary and Westfield College, University of London, London E1 4NS, UK

${ }^{14}$ Technische Hochschule Aachen, III Physikalisches Institut, Sommerfeldstrasse 26-28, D-52056 Aachen, Germany

${ }^{15}$ University College London, London WC1E 6BT, UK

${ }^{16}$ Department of Physics, Schuster Laboratory, The University, Manchester M13 9PL, UK

${ }^{17}$ Department of Physics, University of Maryland, College Park, MD 20742, USA

${ }^{18}$ Laboratoire de Physique Nucléaire, Université de Montréal, Montréal, Quebec H3C 3J7, Canada

${ }^{19}$ University of Oregon, Department of Physics, Eugene OR 97403, USA

${ }^{20}$ Rutherford Appleton Laboratory, Chilton, Didcot, Oxfordshire OX11 0QX, UK

${ }^{21}$ CEA, DAPNIA/SPP, CE-Saclay, F-91191 Gif-sur-Yvette, France

${ }^{22}$ Department of Physics, Technion-Israel Institute of Technology, Haifa 32000, Israel

${ }^{23}$ Department of Physics and Astronomy, Tel Aviv University, Tel Aviv 69978, Israel

${ }^{24}$ International Centre for Elementary Particle Physics and Department of Physics, University of Tokyo, Tokyo 113, and Kobe University, Kobe 657, Japan

${ }^{25}$ Brunel University, Uxbridge, Middlesex UB8 3PH, UK

${ }^{26}$ Particle Physics Department, Weizmann Institute of Science, Rehovot 76100, Israel

${ }^{27}$ Universität Hamburg/DESY, II Institut für Experimental Physik, Notkestrasse 85, D-22607 Hamburg, Germany

${ }^{28}$ University of Victoria, Department of Physics, P O Box 3055, Victoria BC V8W 3P6, Canada 
${ }^{29}$ University of British Columbia, Department of Physics, Vancouver BC V6T 1Z1, Canada

${ }^{30}$ University of Alberta, Department of Physics, Edmonton AB T6G 2J1, Canada

${ }^{31}$ Duke University, Dept of Physics, Durham, NC 27708-0305, USA

${ }^{32}$ Research Institute for Particle and Nuclear Physics, H-1525 Budapest, P O Box 49, Hungary

${ }^{33}$ Institute of Nuclear Research, H-4001 Debrecen, P O Box 51, Hungary

${ }^{a}$ and at TRIUMF, Vancouver, Canada V6T $2 \mathrm{~A} 3$

${ }^{b}$ and Royal Society University Research Fellow

${ }^{c}$ and Institute of Nuclear Research, Debrecen, Hungary

${ }^{d}$ and Department of Experimental Physics, Lajos Kossuth University, Debrecen, Hungary

${ }^{e}$ and Ludwig-Maximilians-Universität, München, Germany 


\section{Introduction}

Parity violation in the weak neutral current results in a polarization of final-state fermion-antifermion pairs produced in $\mathrm{Z}^{0}$ decay with the $\tau$ lepton being the only fundamental fermion whose polarization is experimentally accessible using the detectors at the LEP $\mathrm{e}^{+} \mathrm{e}^{-}$collider. The $\tau$ polarization, $P_{\tau}$, is given by $P_{\tau} \equiv\left(\sigma_{R}-\sigma_{L}\right) /\left(\sigma_{R}+\sigma_{L}\right)$, where $\sigma_{L(R)}$ represents the cross section for producing left(right)-handed $\tau^{-}$leptons ${ }^{1}$. Furthermore, the inequality of the $\mathrm{Z}^{0}$ coupling to left-handed and right-handed initial-state electrons results in a polarization of the $\mathrm{Z}^{0}$ itself, which can be determined by measuring the angular dependence of $P_{\tau}$. For the unpolarized $\mathrm{e}^{+} \mathrm{e}^{-}$ beams at LEP the dependence of $P_{\tau}$ on the angle $\theta_{\tau^{-}}$between the $\mathrm{e}^{-}$beam and the final-state $\tau^{-}$, assuming vector and axial-vector couplings, can be expressed to lowest order as:

$$
P_{\tau}\left(\cos \theta_{\tau^{-}}\right)=\frac{\left\langle P_{\tau}\right\rangle\left(1+\cos ^{2} \theta_{\tau^{-}}\right)+\frac{8}{3} \mathrm{~A}_{\mathrm{pol}}^{\mathrm{FB}} \cos \theta_{\tau^{-}}}{\left(1+\cos ^{2} \theta_{\tau^{-}}\right)+\frac{8}{3} \mathrm{~A}_{\mathrm{FB}} \cos \theta_{\tau^{-}}}
$$

where $\left\langle P_{\tau}\right\rangle$ is the average $\tau$ polarization, $\mathrm{A}_{\mathrm{pol}}^{\mathrm{FB}}$ is the forward-backward polarization asymmetry, which gives the average polarization of the $\mathrm{Z}^{0}$, and $\mathrm{A}_{\mathrm{FB}}$ is the forward-backward asymmetry of the $\tau$-pairs [1]. The forward-backward polarization asymmetry is given by

$$
\mathrm{A}_{\mathrm{pol}}^{\mathrm{FB}} \equiv \frac{\sigma_{R}\left(\cos \theta_{\tau^{-}}>0\right)-\sigma_{L}\left(\cos \theta_{\tau^{-}}>0\right)-\sigma_{R}\left(\cos \theta_{\tau^{-}}<0\right)+\sigma_{L}\left(\cos \theta_{\tau^{-}}<0\right)}{\sigma_{R}+\sigma_{L}} .
$$

Within the Standard Model, the measurement of $\left\langle P_{\tau}\right\rangle$ is directly related to the ratio of the neutral current vector to axial-vector coupling constants for $\tau$ leptons and that of $\mathrm{A}_{\mathrm{pol}}^{\mathrm{FB}}$ to the ratio of the coupling constants for electrons [1] as described in section 6 . Consequently, these measurements provide not only a determination of $\sin ^{2} \theta_{\text {eff }}^{\text {lept }}$, where $\theta_{\text {eff }}^{\text {lept }}$ is the effective electroweak mixing angle for leptons, but also test the hypothesis of lepton universality in the neutral current.

This paper describes a measurement of $\left\langle P_{\tau}\right\rangle$ and $\mathrm{A}_{\mathrm{pol}}^{\mathrm{FB}}$ using the data collected with the OPAL detector at LEP during the period 1990-1994 which corresponds to an integrated luminosity of $117 \mathrm{pb}^{-1}$. It is based on a sample of $89075 \mathrm{e}^{+} \mathrm{e}^{-} \rightarrow \tau^{+} \tau^{-}$candidate events contained within the central region of the detector. The OPAL detector consists of a magnetic spectrometer embedded in electromagnetic (ECAL) and hadronic (HCAL) calorimeters which in turn are surrounded by muon detectors. The detector covers nearly the full solid angle and is described in detail elsewhere [2]. Most of the selected events (90\%) were recorded with the centre-of-mass energy $(\sqrt{\mathrm{s}})$ at the peak of the $\mathrm{Z}^{0}$ resonance and the remainder, referred to as 'off-peak data', recorded at several distinct $\sqrt{\mathrm{s}}$ values within $3 \mathrm{GeV}$ above and below the peak.

The $\tau \rightarrow \rho \nu_{\tau}, \tau \rightarrow \pi(\mathrm{K}) \nu_{\tau}, \tau \rightarrow \mathrm{e} \bar{\nu}_{e} \nu_{\tau}, \tau \rightarrow \mu \bar{\nu}_{\mu} \nu_{\tau}$ and the three-prong $\tau \rightarrow \mathrm{a}_{1} \nu_{\tau}$ decays, representing a combined branching fraction of $83 \%$, are identified and their kinematic properties used to measure the polarization. These new results supersede the measurements reported in reference [3], which were based on an analysis of the 1990-1992 OPAL data sample and did not include the $\tau \rightarrow \mathrm{a}_{1} \nu_{\tau}$ decay channel. The selection criteria for all other channels except the $\tau \rightarrow \mu \bar{\nu}_{\mu} \nu_{\tau}$ channel have been improved in order to reduce the statistical and systematic errors.

The five decay modes do not all have the same sensitivity to the $\tau$ polarization. The $\tau \rightarrow \pi(\mathrm{K}) \nu_{\tau}$ mode has a large sensitivity because it is a two body decay involving a spinless particle, whereas the $\tau \rightarrow \mathbf{e} \bar{\nu}_{e} \nu_{\tau}$ and $\tau \rightarrow \mu \bar{\nu}_{\mu} \nu_{\tau}$ modes have substantially lower sensitivities because

\footnotetext{
${ }^{1}$ By convention, $P_{\tau}=P_{\tau^{-}}$. Note that since, to a very good approximation, the $\tau^{-}$and $\tau^{+}$have opposite helicities at LEP, $P_{\tau^{-}}=-P_{\tau^{+}}$.
} 
the $\tau$ decays to three fermions, two of which are undetected neutrinos. The $\tau \rightarrow \rho \nu_{\tau}$ and $\tau \rightarrow \mathbf{a}_{1} \nu_{\tau}$ decays have reduced sensitivity because they involve spin-1 particles. Much of this sensitivity reduction can be regained by using those kinematic properties of the $\rho$ and $\mathrm{a}_{1}$ decays which are related to the parent's spin orientation. The maximum sensitivity for each decay mode, defined as $\sqrt{N} / \sigma$ where $\sigma$ is the statistical error on the polarization measurement using $N$ events, is given in table 1 which assumes that all the available information in the decay is used with full efficiency ${ }^{2}$. A measure of the weight with which a given decay mode ideally contributes to the overall measurement of the polarization is given by that decay mode's sensitivity squared multiplied by its branching ratio. Normalized ideal weights, which are calculated assuming maximum sensitivity and perfect identification efficiency, for each decay mode are also given in table 1 . As can be seen, the $\tau \rightarrow \rho \nu_{\tau}$ and $\tau \rightarrow \pi(\mathrm{K}) \nu_{\tau}$ channels are expected to dominate the combined polarization measurement. The actual sensitivity achieved in the experiment for the selected event sample is degraded because of inefficiencies in the process of selecting a sample of decays and by the presence of background in the sample.

The extraction of $\left\langle P_{\tau}\right\rangle$ and $\mathrm{A}_{\mathrm{pol}}^{\mathrm{FB}}$ is performed using a global maximum likelihood fit where the data are described by linear combinations of positive and negative helicity distributions in observables appropriate to each $\tau$ decay channel and in the $\tau^{-}$scattering angle, $\theta_{\tau^{-}}$. These distributions are obtained from Monte Carlo simulation. For those events in which both $\tau$ decays have been classified, the analysis explicitly takes into account the $\tau^{+}-\tau^{-}$longitudinal spin correlation and, in so doing, also accounts for experimental correlations between the polarization observables introduced by the $\tau$-pair selection and decay mode identification criteria. The approach is similar to the global fit described in our previous work [3] and has been extended to include all five decay modes.

\begin{tabular}{|l|c|c|c|c|c|}
\hline & $\tau \rightarrow \rho \nu_{\tau}$ & $\tau \rightarrow \pi(\mathrm{K}) \nu_{\tau}$ & $\tau \rightarrow \mathrm{e} \bar{\nu}_{e} \nu_{\tau}$ & $\tau \rightarrow \mu \bar{\nu}_{\mu} \nu_{\tau}$ & $\begin{array}{c}\tau \rightarrow \mathrm{a}_{1} \nu_{\tau} \\
\mathrm{a}_{1}^{ \pm} \rightarrow \pi^{ \pm} \pi^{+} \pi^{-}\end{array}$ \\
\hline Branching ratio & 0.25 & 0.12 & 0.18 & 0.17 & 0.09 \\
Maximum sensitivity & 0.49 & 0.58 & 0.22 & 0.22 & 0.45 \\
Normalized ideal weight & 0.44 & 0.30 & 0.06 & 0.06 & 0.13 \\
\hline
\end{tabular}

Table 1: The branching ratios, maximum sensitivity and normalized ideal weight for the five decay modes used in the analysis. The ideal weight is calculated as the product of the branching ratio and the square of the maximum sensitivity. Presented in the last line of the table is the ideal weight for each channel divided by the sum of the ideal weights of the five channels.

\section{Selection of Tau Decays}

At the first stage of the analysis, a sample of $\mathrm{e}^{+} \mathrm{e}^{-} \rightarrow \tau^{+} \tau^{-}$candidates is selected from which $\tau \rightarrow \rho \nu_{\tau}, \tau \rightarrow \pi(\mathrm{K}) \nu_{\tau}, \tau \rightarrow \mathbf{e} \bar{\nu}_{e} \nu_{\tau}, \tau \rightarrow \mu \bar{\nu}_{\mu} \nu_{\tau}$ and the three-prong $\tau \rightarrow \mathbf{a}_{1} \nu_{\tau}$ decays are identified. The $\tau$-pair sample is selected using the criteria described in our earlier publications $[3,5]$. The general strategy is to identify events characterized by a pair of back-to-back, narrow jets with

\footnotetext{
${ }^{2}$ Note that if information from the $\tau$ direction is available, as discussed in [4], then the sensitivity of the $\tau \rightarrow \mathbf{a}_{1} \nu_{\tau}$ channel can be further improved compared to the figures quoted here.
} 
low particle multiplicity $(\tau$-jet). If the recorded energy is small, the events are required to have unbalanced transverse momentum in order to remove two-photon events. Events with high measured energy which are consistent with being $\mathrm{e}^{+} \mathrm{e}^{-} \rightarrow \mathrm{e}^{+} \mathrm{e}^{-}$or $\mathrm{e}^{+} \mathrm{e}^{-} \rightarrow \mu^{+} \mu^{-}$are also removed. The polar angle of each $\tau$-jet with respect to the direction of the $\mathrm{e}^{-}$beam, $\theta_{j e t}$, is determined using charged tracks and clusters of deposited energy in the ECAL. Events are selected if the average of $\left|\cos \theta_{\text {jet }}\right|$ for the two jets, $\overline{\left|\cos \theta_{\text {jet }}\right|}$, is less than 0.68. Using this selection, a sample of 89075 events is obtained.

The contributions to the selected events from various physics processes are estimated using a number of Monte Carlo data samples. The $\mathrm{e}^{+} \mathrm{e}^{-} \rightarrow \tau^{+} \tau^{-}$signal and $\mathrm{e}^{+} \mathrm{e}^{-} \rightarrow \mu^{+} \mu^{-}$background are both modelled using the KORALZ Monte Carlo generator [6] and the $\mathrm{e}^{+} \mathrm{e}^{-} \rightarrow \mathrm{e}^{+} \mathrm{e}^{-}$background is estimated using the $\mathrm{BABAMC}$ generator [7]. The residual multihadronic background is simulated using the JETSET Monte Carlo [8] with parameters tuned to fit the global event shape distributions of OPAL multihadron data [9]. Contributions from non-resonant t-channel twophoton processes are estimated using the generator described in reference [10]. The response of the OPAL detector to the generated particles in each case is modelled using a simulation program [11] based on the GEANT [12] package. In all cases, the Monte Carlo and real data are treated in an identical manner. Using these Monte Carlo samples, the $\mathrm{e}^{+} \mathrm{e}^{-} \rightarrow \tau^{+} \tau^{-}$selection efficiency is estimated to be $54 \%$ (93\% within the polar angle acceptance) with a purity of $98.3 \%$.

\section{$2.1 \quad \tau \rightarrow \rho \nu_{\tau}$ identification}

Approximately $25 \%$ of $\tau$ leptons decay to a $\tau$ neutrino and a $\rho$ meson, which subsequently decays almost exclusively to a charged and neutral pion. Consequently, the signature of these $\tau \rightarrow \rho \nu_{\tau}$ decays is the presence of a single charged hadron track accompanied by energy deposition in the ECAL, consistent with $\pi^{0}$ decay to two photons, and by a hadronic shower from the charged hadron. A clustering algorithm [13] optimized for $\pi^{0}$ finding in the $\tau$ environment is used to identify $\pi^{0}$ candidates. When there is only one cluster present in the $\tau$-jet which is not associated with the charged track (referred to as a 'neutral cluster'), then it is identified as a $\pi^{0}$ if the cluster energy is more than $1 \mathrm{GeV}$. If there are two neutral clusters present, then the pair is identified as a $\pi^{0}$ if the reconstructed invariant mass of the pair is less than $280 \mathrm{MeV}$. When there are more than two neutral clusters, only the three most energetic neutral clusters are used in the search for a $\pi^{0}$ candidate. At least one pair from among these three clusters must have a reconstructed mass of less than $280 \mathrm{MeV}$ and when more than one pair satisfies this, the pair having a mass closest to $135 \mathrm{MeV}$ is identified as the $\pi^{0}$ candidate. The $\tau \rightarrow \rho \nu_{\tau}$ sample is selected by requiring the presence of a $\pi^{0}$ candidate as defined above and by requiring that the invariant mass of the charged track under a pion hypothesis and the $\pi^{0}$ candidate falls between $0.5 \mathrm{GeV}$ and $2.0 \mathrm{GeV}$. The $\pi^{0}$ requirement implicitly imposes a requirement that the number of neutral clusters be at least one. Reasonable modelling of the lateral distribution of energy deposition in the ECAL is evident from an examination of figure 1a, which shows the data and Monte Carlo distributions of the number of neutral clusters in the one-prong $\tau$ sample from which the $\tau \rightarrow \rho \nu_{\tau}$ selection is made. The $\pi^{ \pm} \pi^{0}$ invariant mass is also adequately modelled in the $\tau \rightarrow \rho \nu_{\tau}$ signal region as can be seen in figure $1 \mathrm{~b}$ in which the distribution of the invariant mass is plotted for both data and Monte Carlo simulation prior to application of the invariant mass requirement. The remaining background from $\tau \rightarrow \mathrm{e} \bar{\nu}_{e} \nu_{\tau}$ and $\tau \rightarrow \pi(\mathrm{K}) \geq 2 \pi^{0} \nu_{\tau}$ decays is further suppressed by requiring that the energy deposited in the ECAL that is associated with the charged track be less than $90 \%$ of the momentum of the track. 
Applying this set of requirements selects $39189 \tau \rightarrow \rho \nu_{\tau}$ candidates from the $\tau$-pair sample. Within the polar-angle acceptance described above, the efficiency is $70 \%$. The background fraction in the $\tau \rightarrow \rho \nu_{\tau}$ sample is $27 \%$ and consists mainly of $\tau \rightarrow \pi(\mathrm{K}) \geq 2 \pi^{0} \nu_{\tau}(17.6 \%)$ and $\tau \rightarrow \pi(\mathrm{K}) \nu_{\tau}(5.3 \%)$ decays. The non- $\tau$ background is estimated to contribute less than $0.4 \%$.

\section{$2.2 \tau \rightarrow \mathrm{e} \bar{\nu}_{e} \nu_{\tau}$ identification}

The $\tau \rightarrow \mathbf{e} \bar{\nu}_{e} \nu_{\tau}$ decays are identified in the sample of $\tau$-jets by employing a likelihood selection technique using observables which provide discrimination between the various single-prong decay channels of the $\tau$ lepton. The Monte Carlo simulation provides normalized distributions for a set of observables, $O_{i}$, for each decay mode. These are subsequently used to calculate for each decay channel $j$, the likelihood, $\ell_{i}^{j}\left(O_{i}\right)$, that the measured $O_{i}$ would be observed. The likelihood that decay mode $j$ produces the measured observables in a given $\tau$-jet is obtained from the product of the likelihoods: $\mathcal{L}(j)=\prod_{i} \ell_{i}^{j}\left(O_{i}\right)$. In order to select decays from mode $k$, a cut is applied to its relative likelihood, $L(k)=\mathcal{L}(k) / \sum_{j} \mathcal{L}(j)$. From this definition, $L(k)$ lies between 0 and 1 . Note that by requiring decays to have large values of $L(k)$, a sample with low background can be obtained at the cost of efficiency for selecting mode $k$ decays. The observables used to form the likelihoods include: the specific energy loss of the charged track as measured in the tracking detector $(\mathrm{dE} / \mathrm{dx})$, the ratio of the energy measured in the ECAL associated with the track to the track momentum, and the number of neutral ECAL clusters in the $\tau$-jet. Observables from the outer detectors such as the number of hits in the muon detectors and number of hits in the HCAL are also used. Before applying the likelihood selection, fiducial requirements are imposed to remove the small fraction of decays having particles entering regions of the detector which are inadequately modelled by the Monte Carlo simulation.

In order to select $\tau \rightarrow \mathrm{e} \bar{\nu}_{e} \nu_{\tau}$ decays three likelihoods are constructed, the likelihood that the decay was $\tau \rightarrow \mathbf{e} \bar{\nu}_{e} \nu_{\tau}, \mathcal{L}\left(\tau \rightarrow \mathbf{e} \bar{\nu}_{e} \nu_{\tau}\right)$, the likelihood the decay was $\tau \rightarrow \mu \bar{\nu}_{\mu} \nu_{\tau}, \mathcal{L}\left(\tau \rightarrow \mu \bar{\nu}_{\mu} \nu_{\tau}\right)$, and the likelihood that the decay was a one-prong hadronic decay, $\mathcal{L}\left(\tau \rightarrow\right.$ hadrons $\left.\nu_{\tau}\right)$. The $\tau \rightarrow \mathrm{e} \bar{\nu}_{e} \nu_{\tau}$ decays are selected by requiring that $L\left(\tau \rightarrow \mathrm{e} \bar{\nu}_{e} \nu_{\tau}\right)>0.5$, where $L\left(\tau \rightarrow \mathrm{e} \bar{\nu}_{e} \nu_{\tau}\right)$ is the relative likelihood that the decay was a $\tau \rightarrow \mathrm{e} \bar{\nu}_{e} \nu_{\tau}$ decay:

$$
L\left(\tau \rightarrow \mathrm{e} \bar{\nu}_{e} \nu_{\tau}\right)=\frac{\mathcal{L}\left(\tau \rightarrow \mathrm{e} \bar{\nu}_{e} \nu_{\tau}\right)}{\mathcal{L}\left(\tau \rightarrow \mathrm{e} \bar{\nu}_{e} \nu_{\tau}\right)+\mathcal{L}\left(\tau \rightarrow \mu \bar{\nu}_{\mu} \nu_{\tau}\right)+\mathcal{L}\left(\tau \rightarrow \text { hadrons } \nu_{\tau}\right)} .
$$

The distribution of $L\left(\tau \rightarrow \mathbf{e} \bar{\nu}_{e} \nu_{\tau}\right)$ for all one-prong decays in the fiducial region is shown in figure 2 a. This selection yields 27352 candidates with an efficiency of $96 \%$ within the fiducial region and a background of $2.7 \%$. Most of the background arises from $\tau \rightarrow \pi(\mathrm{K}) \nu_{\tau}$ decays $(1.2 \%)$ and from $\tau \rightarrow \rho\left(\mathrm{K}^{*}\right) \nu_{\tau}$ decays $(0.8 \%)$. The non- $\tau$ background is estimated to contribute approximately $0.6 \%$.

\section{$2.3 \tau \rightarrow \pi(\mathrm{K}) \nu_{\tau}$ identification}

The $\tau \rightarrow \pi(\mathrm{K}) \nu_{\tau}$ decays are identified in a sample of $\tau$-jets with one charged track having a momentum between $0.05 \mathrm{E}_{\text {beam }}$ and $1.2 \mathrm{E}_{\text {beam }}$, where $\mathrm{E}_{\text {beam }}$ is the beam energy. Starting with this sample, the likelihood selection of $\tau \rightarrow \pi(\mathrm{K}) \nu_{\tau}$ decays proceeds in two stages. The first stage uses the likelihoods defined above to select hadronic tau decays by requiring $L\left(\tau \rightarrow\right.$ hadrons $\left.\nu_{\tau}\right)>0.5$, where

$$
L\left(\tau \rightarrow \text { hadrons } \nu_{\tau}\right)=\frac{\mathcal{L}\left(\tau \rightarrow \text { hadrons } \nu_{\tau}\right)}{\mathcal{L}\left(\tau \rightarrow \mathbf{e} \bar{\nu}_{e} \nu_{\tau}\right)+\mathcal{L}\left(\tau \rightarrow \mu \bar{\nu}_{\mu} \nu_{\tau}\right)+\mathcal{L}\left(\tau \rightarrow \text { hadrons } \nu_{\tau}\right)}
$$


is the relative likelihood that the decay was hadronic. The distribution of $L\left(\tau \rightarrow\right.$ hadrons $\left.\nu_{\tau}\right)$ is shown in figure $2 \mathrm{~b}$.

The second stage selects the $\tau \rightarrow \pi(\mathrm{K}) \nu_{\tau}$ decays from this sample of one-prong hadronic decays. To do this a second set of likelihoods is constructed. The observables used to form these likelihoods are a set of quantities which are sensitive to the presence of neutral pions, the most important of which are the number of neutral clusters, the total ECAL energy, the invariant mass of the charged pion and neutral pion candidates and the invariant mass of the two highest energy neutral clusters (which are assumed to be photons). All ECAL observables used in forming the likelihoods for both the $\tau \rightarrow \mathrm{e} \bar{\nu}_{e} \nu_{\tau}$ and $\tau \rightarrow \pi(\mathrm{K}) \nu_{\tau}$ selections are calculated using the clustering algorithm described in reference [14]. The $\tau \rightarrow \pi(\mathrm{K}) \nu_{\tau}$ decays are selected by requiring $L\left(\tau \rightarrow \pi(\mathrm{K}) \nu_{\tau}\right)>0.5$. In this case, the denominator of the equation analogous to equation 2 is formed by summing over all known hadronic $\tau$ decay modes. The distribution of $L\left(\tau \rightarrow \pi(\mathrm{K}) \nu_{\tau}\right)$ is shown in figure 2c. A total of $18792 \tau \rightarrow \pi(\mathrm{K}) \nu_{\tau}$ candidates is selected with an efficiency of $83 \%$ within the fiducial region with a background level of $19 \%$. Most of the background arises from the $\tau \rightarrow \rho\left(\mathrm{K}^{*}\right) \nu_{\tau}$ mode $(16 \%)$ with the next largest contribution arising from $\tau \rightarrow \mu \bar{\nu}_{\mu} \nu_{\tau}(1 \%)$. The non- $\tau$ background is estimated to contribute approximately $0.1 \%$.

\section{$2.4 \tau \rightarrow \mu \bar{\nu}_{\mu} \nu_{\tau}$ identification}

The $\tau \rightarrow \mu \bar{\nu}_{\mu} \nu_{\tau}$ decays are selected on the basis of cuts applied to single-prong $\tau$-jets using information from the ECAL, HCAL and muon detectors and is essentially unchanged from our previous publications $[3,5]$. Two out of three of these subdetectors are required to register a signal which is consistent with the passage of a minimum ionizing particle associated with the charged track $[3,5]$. This selection has an efficiency of $87 \%$ within the fiducial acceptance and background of $1.9 \%$ where $1.0 \%$ arises from the $\tau \rightarrow \pi(\mathrm{K}) \nu_{\tau}$ decays and the remainder predominantly from the $\mathrm{e}^{+} \mathrm{e}^{-} \rightarrow \mu^{+} \mu^{-}$and two-photon processes. This results in the selection of 23914 decays.

\section{$2.5 \tau \rightarrow \mathbf{a}_{1} \nu_{\tau}$ identification}

For the $\tau \rightarrow \mathrm{a}_{1} \nu_{\tau}$ selection we restrict ourselves to the three-prong mode, which has a branching fraction of $9 \%$. It is assumed that all three-pion decays of the $\tau$ lepton proceed through the $\mathrm{a}_{1}$ [15]. The $\tau \rightarrow \mathrm{a}_{1} \nu_{\tau} \tau$-jet is required to have three charged tracks, none of which is identified as a conversion electron. In order to reduce the contamination from $\tau \rightarrow 3 h \geq 1 \pi^{0} \nu_{\tau}$ decays ${ }^{3}$, the ratio of the total ECAL energy measured in the $\tau$-jet to the sum of the momenta of the three tracks is required to be less than $\mathbf{0 . 6 0}$. The distribution of this ratio in the three-prong sample after removing the conversion electrons is shown in figure $3 \mathrm{a}$ for both the data and Monte Carlo simulation. Reasonable modelling of this variable is evident from this figure. The number of selected $\tau \rightarrow \mathrm{a}_{1} \nu_{\tau}$ candidates is 13792 . The selection efficiency is $66 \%$ and the background is $25 \%$, most of which is from $\tau \rightarrow 3 h \geq 1 \pi^{0} \nu_{\tau}$ decays ${ }^{4}$.

\footnotetext{
${ }^{3}$ The symbol $h$ refers to a charged pion or kaon.

${ }^{4} \mathrm{~A}$ branching ratio of $5.1 \%$ has been used for the mode $\tau \rightarrow 3 h \geq 1 \pi^{0} \nu_{\tau}$, using the convention which excludes the $\mathrm{K}_{\mathrm{S}}^{0}$ decays.
} 


\section{$3 \quad$ Fitting Method}

In order to measure $\left\langle P_{\tau}\right\rangle$ and $\mathrm{A}_{\mathrm{pol}}^{\mathrm{FB}}$, distributions of kinematic variables of the $\tau$ decay products which depend on the $\tau$ helicity are used. These variables, as well as their distributions, depend on the decay mode used ${ }^{5}$. For $\tau \rightarrow \mathrm{e} \bar{\nu}_{e} \nu_{\tau}, \tau \rightarrow \mu \bar{\nu}_{\mu} \nu_{\tau}$ and $\tau \rightarrow \pi(\mathrm{K}) \nu_{\tau}$ decays, the relevant kinematic variable, $x$, is the charged particle energy scaled by the beam energy. For $\tau \rightarrow \mathbf{e} \bar{\nu}_{e} \nu_{\tau}$ decays, the energy measured in the ECAL associated with the $\tau$-jet is used, whereas for $\tau \rightarrow \mu \bar{\nu}_{\mu} \nu_{\tau}$ and $\tau \rightarrow \pi(\mathrm{K}) \nu_{\tau}$ decays, the energy is determined using the momentum of the charged particle measured in the central tracking detector.

For $\tau \rightarrow \rho \nu_{\tau}$ decays two variables are used: $\theta^{*}$, the angle of the $\rho$ momentum relative to the $\tau$ flight direction in the $\tau$ rest frame, and $\psi$, the angle of the charged pion relative to the $\rho$ flight direction in the $\rho$ rest frame. This spin-analysis of the $\rho$ decay recuperates most of the sensitivity which would otherwise be lost as a consequence of the angular momentum carried off by the spin of the $\rho$.

The case of the $\tau \rightarrow \mathrm{a}_{1} \nu_{\tau}$ decay is more complicated because the $\mathrm{a}_{1}$ decays into three pions. Six observables are used in order to maximize the sensitivity in the $\tau \rightarrow \mathbf{a}_{1} \nu_{\tau}$ channel: the angle between the $\mathrm{a}_{1}$ and $\tau$ momenta in the $\tau$ rest frame, the angle between the perpendicular to the $a_{1}$ decay plane and the $a_{1}$ flight direction in the rest frame of the $a_{1}$, the angle in the $a_{1}$ rest frame between the unlike-sign pion momentum in the $a_{1}$ rest frame and the $a_{1}$ flight direction projected into the $a_{1}$ decay plane, the $3 \pi$-invariant mass, and the two $\pi^{+} \pi^{-}$mass combinations present in the $\mathrm{a}_{1}^{ \pm} \rightarrow \pi^{ \pm} \pi^{+} \pi^{-}$decay. The distribution of the invariant mass of the three charged particles assuming them all to be pions, shown in figure $3 \mathrm{~b}$, demonstrates that agreement between the data and simulation of this quantity is reasonable. The Monte Carlo distribution depends on the mass and width of the $a_{1}$ as defined within the framework of a particular model of $\tau \rightarrow \mathrm{a}_{1} \nu_{\tau}$ decay[16] and allowance in the assignment of systematic errors must be made for $\tau \rightarrow \mathrm{a}_{1} \nu_{\tau}$ model dependence. The six observables are converted into a single optimum variable, $\omega$, with no polarization sensitivity loss [4]. The variable $\omega$ is defined by $\omega=\left(R_{R}-R_{L}\right) /\left(R_{R}+R_{L}\right)$ where $R_{R}$ and $R_{L}$ are the population densities of right-handed and left-handed $\tau$ lepton decays, respectively, which are functions of the six variables mentioned above.

The joint distributions of the $\tau$-pair production and decay can be expressed as:

$$
\begin{aligned}
\frac{d^{3} \sigma_{i j}}{d \cos \theta_{\tau^{-}} d x_{i} d x_{j}}= & \frac{3}{16} \sigma_{i j} \sum_{\lambda= \pm 1}\left[\left(1+\cos ^{2} \theta_{\tau^{-}}+\frac{8}{3} \mathrm{~A}_{\mathrm{FB}} \cos \theta_{\tau^{-}}\right)+\right. \\
& \left.\lambda\left(\left\langle P_{\tau}\right\rangle\left(1+\cos ^{2} \theta_{\tau^{-}}\right)+\frac{8}{3} \mathrm{~A}_{\mathrm{pol}}^{\mathrm{FB}} \cos \theta_{\tau^{-}}\right)\right] \times \\
& {\left[F_{i}\left(x_{i},\left|\cos \theta_{\tau^{-}}\right|\right)+\lambda G_{i}\left(x_{i},\left|\cos \theta_{\tau^{-}}\right|\right)\right]\left[F_{j}\left(x_{j},\left|\cos \theta_{\tau^{-}}\right|\right)+\lambda G_{j}\left(x_{j},\left|\cos \theta_{\tau^{-}}\right|\right)\right] }
\end{aligned}
$$

where $\sigma_{i j}$ is the cross-section to produce an $\mathrm{e}^{+} \mathrm{e}^{-} \rightarrow \tau^{+} \tau^{-}$event in which one $\tau$ decays via channel $i$ and the other via channel $j$. The first two lines of equation 3 refer to the production of the $\tau$-pairs and the third line to the $\tau$ decays. The summation over $\lambda$ indicates that the summation is over positive and negative helicities. The decay distributions for right-handed $\tau$ leptons are given by $F_{i}+G_{i}$ whereas the decay distributions for left-handed taus are given by $F_{i}-G_{i}$. Note that $F_{i}$ and $G_{i}$ represent functions of $x_{i}$, which represents the pertinent kinematic variable(s) for channel $i$, and $\left|\cos \theta_{\tau^{-}}\right|$after including the effects of the decay mode identification procedure, detector response and radiation. For the $\tau \rightarrow \rho \nu_{\tau}$ decays, $x$ represents the pair of variables, $\cos \theta^{*}$ and $\cos \psi$, while for $\tau \rightarrow \mathrm{a}_{1} \nu_{\tau}$ decays $x$ represents the $\omega$ variable. The

\footnotetext{
${ }^{5}$ Note that the distributions are the same for the $\tau^{+}$and $\tau^{-}$provided that $\left\langle P_{\tau}\right\rangle$ is the $\tau^{-}$helicity.
} 
simulation of the observables used in the analysis is checked, and corrected if necessary, using various control samples as discussed below. Expression 3 includes the correlation between the decay distributions of the two $\tau$ leptons when analysing events in which both $\tau$ decay channels are identified. There is some probability that a decay is identified in more than one channel but this is negligible except in the case where simultaneous $\tau \rightarrow \pi(\mathrm{K}) \nu_{\tau}$ and $\tau \rightarrow \rho \nu_{\tau}$ assignments are made. In this case, the sensitivities of the two modes are similar, and roughly half the overlapping sample is predicted by the Monte Carlo to arise from each mode. In order to minimize any possible bias, the analysis is performed twice, when all ambiguous decays are assigned to one channel and then to the other, and the average $\left\langle P_{\tau}\right\rangle$ and $\mathrm{A}_{\text {pol }}^{\mathrm{FB}}$ quoted.

A binned maximum likelihood fit is performed to extract simultaneously $\left\langle P_{\tau}\right\rangle$ and $\mathrm{A}_{\mathrm{pol}}^{\mathrm{FB}}$ by fitting the linear combination of the positive and negative helicity Monte Carlo distributions to the data. This was favoured over the unbinned likelihood fit of reference [3] because it avoids the need to determine parametrizations of $F$ and $G$, which becomes particularly problematic for the two-dimensional distributions used for the $\tau \rightarrow \rho \nu_{\tau}$ channel. The values of $x_{i}, x_{j}$ and $\cos \theta_{\tau^{-}}$for each event are calculated and a histogram binned ${ }^{6}$ in $x_{i}, x_{j}$ and $\cos \theta_{\tau^{-}}$is then filled for each $\sqrt{\mathrm{s}}$. A value for $\cos \theta_{\tau^{-}}$of the event is determined from $\overline{\left|\cos \theta_{\text {jet }}\right|}$ and the sign of the charge of the identified $\tau$ decay. A separate set of histograms exists for each combination of decay channel pairs. If only one $\tau$ decay is identified, then only bins in $x_{i}$ and $\cos \theta_{\tau^{-}}$are filled. The same procedure is performed for the Monte Carlo with a separate set of histograms filled for the positive and negative helicity $\tau$ leptons binned in $x_{i}, x_{j}$ and $\left|\cos \theta_{\tau^{-}}\right|$. This provides the product $\left[F_{i}+\lambda G_{i}\right]\left[F_{j}+\lambda G_{j}\right]$ as a function of $\left|\cos \theta_{\tau^{-}}\right|$in the Monte Carlo, which uses the fact that the detector is symmetric in $\cos \theta_{\tau^{-}}$. As a consequence, the forward and backward hemispheres use the same Monte Carlo sample. Therefore, the correlations in the Monte Carlo samples result in a reduced Monte Carlo statistical error on $\mathrm{A}_{\mathrm{pol}}^{\mathrm{FB}}$.

The Monte Carlo statistics are taken into account in the likelihood fit in the manner described in reference [17]. In order to identify the contribution to the total error arising from the data statistical error only, a second fit is performed which does not take into account the Monte Carlo statistical errors. The Monte Carlo statistical error is taken to be the quadratic difference between the error from the fit taking into account both data and Monte Carlo statistical errors and that from the fit which only takes the data errors into account.

The effects on the measured polarization arising from misidentified $\tau$ decays are modelled by the Monte Carlo simulation. The helicity dependence of the misidentified decays is automatically taken into account in the product $\left[F_{i}+\lambda G_{i}\right]\left[F_{j}+\lambda G_{j}\right]$. Contributions from the small non- $\tau$ background are estimated using Monte Carlo simulations of distributions in the relevent kinematic variables. As there is no helicity dependence in this background, these distributions are added to the linear combination of the right-handed and left-handed $\tau$ decay Monte Carlo distributions to form the complete reference distributions used in the fit.

The fit also depends on $\mathrm{A}_{\mathrm{FB}}$ for which the measured value in the $\mathrm{Z}^{0} \rightarrow \tau^{+} \tau^{-}$channel [18] at the appropriate $\sqrt{\mathrm{s}}$ is used. Separate distributions for the different values of $\sqrt{\mathrm{s}}$ are used in order to account for the $A_{\mathrm{FB}}$ dependence but a single fit for $\left\langle P_{\tau}\right\rangle$ and $\mathrm{A}_{\mathrm{pol}}^{\mathrm{FB}}$ is performed. Although there are potential dependences of the observables in the analysis on the exact value of $\sqrt{\mathrm{s}}$ at which the data were collected, the use of beam-energy normalized observables renders the analysis relatively insensitive to such effects. However, in order to further reduce any such dependences, the data collected with $\sqrt{\mathrm{s}}$ below $90.7 \mathrm{GeV}$ are fitted using reference distributions from Monte Carlo samples generated at $\sqrt{\mathrm{s}}=89.5 \mathrm{GeV}$ while data collected with $\sqrt{\mathrm{s}}$ above

\footnotetext{
${ }^{6}$ There are six bins in $\cos \theta_{\tau^{-}}$, twelve bins in $x$ for the $\tau \rightarrow \pi(\mathrm{K}) \nu_{\tau}, \tau \rightarrow \mathrm{e} \bar{\nu}_{e} \nu_{\tau}$, and $\tau \rightarrow \mu \bar{\nu}_{\mu} \nu_{\tau}$ modes, ten bins in $\omega$ for the $\tau \rightarrow \mathbf{a}_{1} \nu_{\tau}$ and five-by-five bins in $\cos \theta^{*}$ and $\cos \psi$ for the $\tau \rightarrow \rho \nu_{\tau}$ decays.
} 
91.7 GeV are analysed using samples generated at $\sqrt{\mathrm{s}}=93.0 \mathrm{GeV}$. Most of the off-peak data were collected with values of $\sqrt{\mathrm{s}}$ within $0.1 \mathrm{GeV}$ of the values used in the Monte Carlo generation. The polarization asymmetries quoted below are for $\sqrt{s}=91.18 \mathrm{GeV}$, and therefore a small (less than $0.1 \%$ ) shift, obtained from ZFITTER [19], is applied to the fitted parameters in order to correct for the $\sqrt{\mathrm{s}}$ dependence.

The global fit technique has been checked with independent fits to each channel, the results of which are presented in table 2. The weighted average of $\left\langle P_{\tau}\right\rangle$ of these fit results differs from that obtained from the global fit by $0.7 \%$, which is approximately half the total error. This difference is consistent with expected statistical fluctuations in the difference between results obtained using a fit which takes into account correlations and a weighted average which does not.

\begin{tabular}{|l|c|c|c|c|c|}
\hline & $\tau \rightarrow \rho \nu_{\tau}$ & $\tau \rightarrow \pi(\mathrm{K}) \nu_{\tau}$ & $\tau \rightarrow \mathrm{e} \bar{\nu}_{e} \nu_{\tau}$ & $\tau \rightarrow \mu \bar{\nu}_{\mu} \nu_{\tau}$ & $\tau \rightarrow \mathrm{a}_{1} \nu_{\tau}$ \\
\hline Sample size & 39189 & 18792 & 27352 & 23914 & 13792 \\
Efficiency & $70 \%$ & $83 \%$ & $96 \%$ & $87 \%$ & $66 \%$ \\
Background & $27 \%$ & $19 \%$ & $2.7 \%$ & $1.9 \%$ & $25 \%$ \\
\hline$\left\langle P_{\tau}\right\rangle(\%)$ & $-11.6 \pm 1.3$ & $-11.7 \pm 1.4$ & $-16.1 \pm 3.3$ & $-13.8 \pm 3.3$ & $-15.1 \pm 3.7$ \\
$\mathrm{~A}_{\text {pol }}^{\mathrm{FB}}(\%)$ & $-9.6 \pm 1.6$ & $-8.8 \pm 1.6$ & $-11.0 \pm 3.8$ & $-14.3 \pm 3.8$ & $-7.6 \pm 4.2$ \\
\hline
\end{tabular}

Table 2: The number of decays in the sample, selection efficiency within the fiducial acceptance and background for each decay mode analysed. Results of independent fits for the individual decay modes are also presented where the error quoted represents that arising from the data statistics only. The measurements from the individual channels are correlated and therefore should not be combined in a simple average.

As a demonstration of the validity of the Monte Carlo simulation of the kinematic variables used in the fit, the one-dimensional distributions of the relevant kinematic variables for the $\tau \rightarrow \mathrm{e} \bar{\nu}_{e} \nu_{\tau}, \tau \rightarrow \mu \bar{\nu}_{\mu} \nu_{\tau}, \tau \rightarrow \pi(\mathrm{K}) \nu_{\tau}$ and $\tau \rightarrow \mathrm{a}_{1} \nu_{\tau}$ channels combining data from all $\cos \theta_{\tau^{-}}$bins are shown in figure 4 for both the data and Monte Carlo. Also shown are the Monte Carlo distributions of the variables for positive and negative helicity $\tau$ lepton decays and their sum including non- $\tau$ background, assuming the value of $\left\langle P_{\tau}\right\rangle$ reported below. The analogous information for the $\tau \rightarrow \rho \nu_{\tau}$ data is displayed in figure 5 showing the reconstructed $\cos \theta^{*}$ distribution in four bins of $\cos \psi$. The enhancement in the distribution in figure $5(\mathrm{a})$ at $\cos \theta^{*}=-1$ is a consequence of the underlying physics distribution. In figure $5(\mathrm{~d})$ the enhancement at $\cos \theta^{*}=1$ is dominated by background from $\tau \rightarrow \pi(\mathrm{K}) \nu_{\tau}$ decays. In addition, there is enhancement in these distributions at $\cos \theta^{*}= \pm 1$ from overflow, there being nothing to constrain the reconstructed values of $\cos \theta^{* 7}$ to lie within \pm 1 . There is good agreement between the data and Monte Carlo expectations in all distributions.

As a further check on the validity of the fit, the results of fits for $P_{\tau}$ performed independently in six bins of $\cos \theta_{\tau^{-}}$are shown in figure 6 . For the fit in a particular $\cos \theta_{\tau^{-}}$bin, an expression analogous to that shown in equation 3 is used in which $\left(\left\langle P_{\tau}\right\rangle\left(1+\cos ^{2} \theta_{\tau^{-}}\right)+\frac{8}{3} \mathrm{~A}_{\mathrm{pol}}^{\mathrm{FB}} \cos \theta_{\tau^{-}}\right)$in the second line of equation 3 is replaced by $\left(P_{\tau}\left(1+\cos ^{2} \theta_{\tau^{-}}+\frac{8}{3} \mathrm{~A}_{\mathrm{FB}} \cos \theta_{\tau^{-}}\right)\right)$. This substitution uses equation 1 . Overlaying these points is a curve which represents the expectation value

\footnotetext{
${ }^{7}$ The value of $\cos \theta^{*}$ is reconstructed in terms of the $\tau$ mass, reconstructed $\rho$-meson mass and the energies of the $\rho$ and $\tau$ using the expression $\cos \theta^{*}=\left[M_{\tau}^{2}\left(2 E_{\rho} / E_{\tau}-1\right)-M_{\rho}^{2}\right] /\left[M_{\tau}^{2}-M_{\rho}^{2}\right]$ where $E_{\tau}$ is assumed to be $E_{\text {beam }}$.
} 
of $P_{\tau}$ as a function of $\cos \theta_{\tau^{-}}$using the values of the $\left\langle P_{\tau}\right\rangle$ and $\mathrm{A}_{\mathrm{pol}}^{\mathrm{FB}}$ from the full maximum likelihood fit given below and equation 1. The results of the six independent fits are in good agreement with the expectations from the global fit: the $\chi^{2}$ is 4.9 for four degrees of freedom when comparing the six values of $\mathrm{P}_{\tau}$ to the expected value from the global fit where only the data and Monte Carlo statistics are included in calculating the $\chi^{2}$.

\section{Detector Systematic Errors}

Because the Monte Carlo simulation provides the positive and negative helicity reference distributions in the fit, it is necessary that the detector response be accurately modelled. High purity control samples of muons with momenta of approximately $45 \mathrm{GeV}$ from $\mathrm{e}^{+} \mathrm{e}^{-} \rightarrow \mu^{+} \mu^{-}$ events are used to determine corrections to the simulation of the momentum scale and resolution of the central tracking detector. The systematic uncertainties of these corrections yield a momentum scale uncertainty of $0.2 \%$. These corrections were cross checked at lower energies using the transverse momentum distributions in $\mathrm{e}^{+} \mathrm{e}^{-} \rightarrow \mathrm{e}^{+} \mathrm{e}^{-} \mu^{+} \mu^{-}$two-photon processes. Studies of measurements of the masses of the $\mathrm{K}_{\mathrm{S}}^{0}, \mathrm{D}^{0}$ and $\mathrm{D}^{+}$from $\mathrm{K}_{\mathrm{S}}^{0} \rightarrow \pi^{+} \pi^{-}, \mathrm{D}^{0} \rightarrow \mathrm{K}^{-} \pi^{+}$and $\mathrm{D}^{+} \rightarrow \mathrm{K}^{-} \pi^{+} \pi^{+}$provide additional checks of the momentum scale at the lower energies. Pure samples of electrons with energies of approximately $45 \mathrm{GeV}$ from $\mathrm{e}^{+} \mathrm{e}^{-} \rightarrow \mathrm{e}^{+} \mathrm{e}^{-}$events are used to determine corrections to the simulation of the energy scale and resolution of the ECAL. These corrections were cross checked at lower energies using the ratio of the deposited energy to measured momentum for electrons in $\mathrm{e}^{+} \mathbf{e}^{-} \rightarrow \mathbf{e}^{+} \mathbf{e}^{-} \mathbf{e}^{+} \mathbf{e}^{-}$two-photon processes and in pure $\tau \rightarrow \mathrm{e} \bar{\nu}_{e} \nu_{\tau}$ samples. An uncertainty of $0.3 \%$ on the ECAL energy scale is estimated from these studies. The one standard deviation errors on the energy scale $( \pm 0.3 \%)$ and momentum scale $( \pm 0.2 \%)$ are used in assessing the systematic errors on $\left\langle P_{\tau}\right\rangle$ and $\mathrm{A}_{\mathrm{pol}}^{\mathrm{FB}}$ from an analysis using rescaled energy and momenta which takes into account the correlations between channels. In a similar manner, systematic errors associated with uncertainties in the parameters used to describe the resolutions of the ECAL and tracking detector resolution are also assigned.

High purity muon and $\tau \rightarrow \rho \nu_{\tau}$ samples are used to correct the modelling of the response of the HCAL and muon chambers to muons and hadrons. Variation of the magnitude of these corrections is used to assess the systematic error on $\left\langle P_{\tau}\right\rangle$ and $\mathrm{A}_{\mathrm{pol}}^{\mathrm{FB}}$ associated with this modelling. Correct modelling of the $\mathrm{dE} / \mathrm{dx}$ measurement is achieved by studying the response of the tracking detector to high purity $\tau \rightarrow \mathrm{e} \bar{\nu}_{e} \nu_{\tau}$ and $\tau \rightarrow \mu \bar{\nu}_{\mu} \nu_{\tau}$ samples selected without using $\mathrm{dE} / \mathrm{dx}$ information. The corrections applied to the $\mathrm{dE} / \mathrm{dx}$ simulation are changed in order to assess the sensitivity of $\left\langle P_{\tau}\right\rangle$ and $\mathrm{A}_{\mathrm{pol}}^{\mathrm{FB}}$ to this modelling. The effects of uncertainties in the amount of material in the central detector, which potentially affects the photon conversion background in the $a_{1}$ channel, were studied and found to have a negligible influence on the polarization measurement.

The uncertainty in the modelling of the lateral spread of the electromagnetic and hadronic showers contributes significantly to the overall systematic error. This is particularly relevant for the separation of the $\tau \rightarrow \pi(\mathrm{K}) \nu_{\tau}$ and $\tau \rightarrow \rho \nu_{\tau}$ samples. The influence of these uncertainties on the polarization measurement is estimated by varying the thresholds in the cluster definitions for the simulation, by studying the stability of the results from the likelihood selection when excluding individual observables related to showering, and from the cluster position resolution uncertainties. Further checks of this class of systematic error in the $\tau \rightarrow \rho \nu_{\tau}$ analysis were available from cut variation studies, which confirmed the magnitude of the assigned errors. The ECAL cluster position resolution is also sensitive to the lateral shower spread in the ECAL. 
The $\pi^{0}$ invariant mass distribution and a control sample of electrons are used to improve the modelling of the ECAL cluster position resolution and to assign uncertainties to this modelling by varying the magnitude of the corrections applied to the simulation. In addition, part of the ECAL hadronic shower uncertainty in the global analysis is estimated from the different polarization results arising from assigning the doubly-identified $\tau \rightarrow \pi(\mathrm{K}) \nu_{\tau}$ and $\tau \rightarrow \rho \nu_{\tau}$ decays to the $\pi$ and $\rho$ channels. As the average of these values is quoted, the associated systematic error, which is equal to half the difference $(0.2 \%)$, is included in the systematic error of the global result. There is also a potential systematic error on $A_{\mathrm{pol}}^{\mathrm{FB}}$ related to charge mis-assignment, which in OPAL is negligible. The contributions to the uncertainty on $\left\langle P_{\tau}\right\rangle$ and $A_{\text {pol }}^{\mathrm{FB}}$ from these various sources are shown in table 3 .

\begin{tabular}{|c|c|c|c|c|c|c|c|c|c|c|c|c|}
\hline & \multicolumn{12}{|c|}{$\Delta\left\langle P_{\tau}\right\rangle$ and $\Delta \mathrm{A}_{\mathrm{pol}}^{\mathrm{FB}}(\%)$} \\
\hline & \multicolumn{2}{|c|}{$\rho$} & \multicolumn{2}{|c|}{$\pi(\mathrm{K})$} & \multicolumn{2}{|c|}{$\mathrm{e}$} & \multicolumn{2}{|c|}{$\mu$} & \multicolumn{2}{|c|}{$a_{1}$} & \multicolumn{2}{|c|}{ Global fit } \\
\hline Momentum scale/resolution & 0.5 & - & 0.7 & 0.1 & 1.3 & - & 1.4 & 0.1 & 0.2 & 0.9 & 0.4 & 0.1 \\
\hline ECAL scale/resolution & 0.3 & 0.1 & 0.1 & 0.2 & 2.0 & 0.1 & - & - & 0.2 & 0.6 & 0.2 & 0.1 \\
\hline HCAL/MUON modelling & - & - & 0.3 & 0.1 & 0.6 & 0.1 & 0.8 & 0.1 & - & - & 0.2 & - \\
\hline $\mathrm{dE} / \mathrm{dx}$ errors & - & - & 0.2 & - & 0.3 & - & - & - & - & - & 0.1 & - \\
\hline Shower modelling in ECAL & 0.5 & 0.2 & 0.5 & 0.2 & 0.5 & 0.4 & - & - & 1.2 & 0.6 & 0.5 & 0.2 \\
\hline Branching ratios & 0.2 & - & 0.4 & - & 0.1 & - & 0.1 & - & 0.6 & 0.2 & 0.2 & - \\
\hline$\tau \rightarrow \mathrm{a}_{1} \nu_{\tau}$ modelling & 0.4 & 0.4 & - & - & - & - & - & - & 1.3 & 0.3 & 0.3 & 0.2 \\
\hline$\tau \rightarrow 3 h \geq 1 \pi^{0} \nu_{\tau}$ modelling & - & - & - & - & - & - & - & - & 1.9 & 1.2 & 0.1 & 0.1 \\
\hline$A_{F B}$ & - & 0.1 & - & - & - & 0.1 & 0.1 & 0.1 & - & - & - & 0.1 \\
\hline Decay radiation & 0.1 & - & - & - & - & - & - & - & - & - & - & - \\
\hline Monte Carlo statistics & 0.6 & 0.1 & 0.7 & 0.1 & 1.5 & 0.6 & 1.5 & 0.5 & 1.5 & 0.5 & 0.5 & 0.3 \\
\hline total & 1.1 & 0.5 & 1.2 & 0.3 & 2.9 & 0.7 & 2.2 & 0.5 & 3.1 & 1.8 & 0.9 & 0.4 \\
\hline
\end{tabular}

Table 3: Tabulation of systematic errors contributing to $\left\langle P_{\tau}\right\rangle$ and $\mathrm{A}_{\mathrm{pol}}^{\mathrm{FB}}$ in percent for each of the five decay modes analysed and the global fit. In each column the error on $\left\langle P_{\tau}\right\rangle$ is given first followed by that on $\mathrm{A}_{\mathrm{pol}}^{\mathrm{FB}}$. A dash indicates that the listed effect contributes less than $0.05 \%$ to the systematic error.

\section{$5 \quad$ Tau Production and Decay Systematic Errors}

Another class of systematic uncertainties relates to our knowledge of $\tau$ production and decay. In this category are the errors on measured branching ratios of the different $\tau$ decay modes. The branching ratios used are obtained from an average of the uncorrelated measurements in references [20-24]. The error on $\left\langle P_{\tau}\right\rangle$ and $A_{\mathrm{pol}}^{\mathrm{FB}}$ associated with the uncertainty of each branching ratio is estimated by varying the value used in the global analysis by \pm 1 standard deviation about its average value.

The uncertainty in the modelling of the $\tau \rightarrow \mathbf{a}_{1} \nu_{\tau}$ decay introduces systematic errors both in the $\tau \rightarrow \mathrm{a}_{1} \nu_{\tau}$ channel and in the $\tau \rightarrow \rho \nu_{\tau}$ channel where the $\tau \rightarrow \mathrm{a}_{1} \nu_{\tau}$ decays represent $16 \%$ of the selected decays. Two contributions to the $\tau \rightarrow \mathrm{a}_{1} \nu_{\tau}$ modelling uncertainty are considered: one being the uncertainty of the mass and width of the $a_{1}$ as obtained from reference [25] and the other obtained by comparing two independent theoretical treatments of the $\tau \rightarrow \mathrm{a}_{1} \nu_{\tau}$ decay $[16$, 
26]. In the $\tau \rightarrow \rho \nu_{\tau}$ analysis consideration was given to a fit using a single optimal variable [4], $\omega$, analogous to that used in the $\tau \rightarrow \mathrm{a}_{1} \nu_{\tau}$ analysis, instead of the two-dimensional fit. The results obtained using that variable were found to be significantly more sensitive to the modelling of the $a_{1}$ background and therefore the two-dimensional analysis is preferred for the levels of $\tau \rightarrow \mathrm{a}_{1} \nu_{\tau}$ background present in our sample.

In addition to the $\tau \rightarrow \mathrm{a}_{1} \nu_{\tau}$ modelling uncertainty, the modelling of the $\tau \rightarrow 3 h \geq 1 \pi^{0} \nu_{\tau}$ decays introduces an independent uncertainty in the analysis of the $\tau \rightarrow \mathrm{a}_{1} \nu_{\tau}$ channel. This is studied by varying the selection requirement on the ratio of the total ECAL energy to the summed track momenta. As the measured quantities used in this ratio are adequately modelled in terms of detector response, the resulting variation in the polarization is predominantly sensitive to the modelling of the $\tau \rightarrow 3 h \geq 1 \pi^{0} \nu_{\tau}$ decay and the excursion of the extracted polarization asymmetries from the nominal values are assigned as errors.

Two smaller sources of error also fall into this general category of systematic error: the error associated with the measured value of $\mathrm{A}_{\mathrm{FB}}$ for $\mathrm{e}^{+} \mathrm{e}^{-} \rightarrow \boldsymbol{\tau}^{+} \tau^{-}$events which is obtained from reference [18] and the uncertainty of the simulation of radiation in the decay of the $\tau$ which is treated in the same manner as is described in reference [3].

The contributions arising from the various systematic errors are summarized in table 3 for each of the independent analyses and for the global analysis which takes into account the correlations between channels.

\section{$6 \quad$ Results and Discussion}

The results of the global fit are:

$$
\begin{aligned}
& \left\langle P_{\tau}\right\rangle=(-13.0 \pm 0.9 \pm 0.9) \% \\
& \mathrm{~A}_{\mathrm{pol}}^{\mathrm{FB}}=(-9.4 \pm 1.0 \pm 0.4) \%
\end{aligned}
$$

where the first error is statistical and the second systematic. Both the statistical and systematic correlations between the two parameters obtained from the fit are found to be less than 0.01 . From these values of $\left\langle P_{\tau}\right\rangle$ and $\mathrm{A}_{\mathrm{pol}}^{\mathrm{FB}}$ together with equation 1, a measurement of $P_{\tau}\left(\cos \theta_{\tau^{-}}\right)$is obtained with a central value as plotted in figure 6 and with a total error ranging from $1.3 \%$ at $\cos \theta_{\tau^{-}}=0$ to $1.8 \%$ at the edge of the geometrical acceptance of the analysis, $\left|\cos \theta_{\tau^{-}}\right|=0.68$.

These measurements are consistent with our previous measurement [3] but with an error that has been reduced by a factor of two. The results are also consistent with those published by other LEP collaborations [27, 28, 29].

The Standard Model gives predictions for $\left\langle P_{\tau}\right\rangle$ and $\mathrm{A}_{\mathrm{pol}}^{\mathrm{FB}}$ in terms of $\sqrt{\mathrm{s}}$, the mass and width of the $\mathrm{Z}^{0}$, and its vector $\left(g_{v}^{\ell}\right)$ and axial-vector $\left(g_{a}^{\ell}\right)$ couplings to the electron and the $\tau$ lepton. In the improved Born approximation [30], which accounts for the most significant weak radiative corrections, and neglecting the contributions of the intermediate photon, photon- $Z^{0}$ interference and other photonic radiative corrections, the Standard Model predicts for $\sqrt{\mathrm{s}}=\mathrm{M}_{\mathrm{Z}}$ :

$$
\left\langle P_{\tau}\right\rangle=-\mathcal{A}_{\tau} \quad \text { and } \quad \mathrm{A}_{\mathrm{pol}}^{\mathrm{FB}}=-\frac{3}{4} \mathcal{A}_{\mathrm{e}}
$$

where

$$
\mathcal{A}_{\ell} \equiv \frac{2 \hat{\mathrm{g}}_{v}^{\ell} / \hat{\mathrm{g}}_{a}^{\ell}}{1+\left(\hat{\mathrm{g}}_{v}^{\ell} / \hat{\mathrm{g}}_{a}^{\ell}\right)^{2}} .
$$


The ratio between the effective vector and axial-vector couplings [30], $\hat{\mathrm{g}}_{v}^{\ell} / \hat{\mathrm{g}}_{a}^{\ell}$, is related to the effective electroweak mixing angle by:

$$
\hat{\mathrm{g}}_{v}^{\ell} / \hat{\mathrm{g}}_{a}^{\ell}=1-4 \sin ^{2} \theta_{\mathrm{eff}}^{\mathrm{lept}} .
$$

Expressing our measurements of $\left\langle P_{\tau}\right\rangle$ and $\mathrm{A}_{\mathrm{pol}}^{\mathrm{FB}}$ in terms of $\mathcal{A}_{\tau}$ and $\mathcal{A}_{\mathrm{e}}$, and using ZFITTER $[19]$ to correct for the effects of the photon propagator, photon- $Z^{0}$ interference and photonic radiative corrections, gives:

$$
\begin{aligned}
& \mathcal{A}_{\tau}=0.134 \pm 0.009 \pm 0.010 \\
& \mathcal{A}_{\mathbf{e}}=0.129 \pm 0.014 \pm 0.005
\end{aligned}
$$

Within the context of the Standard Model these results can be interpreted as measurements of:

$$
\begin{aligned}
& \hat{\mathrm{g}}_{v}^{\tau} / \hat{\mathrm{g}}_{a}^{\tau}=0.0674 \pm 0.0066 \\
& \hat{\mathrm{g}}_{v}^{e} / \hat{\mathrm{g}}_{a}^{e}=0.0650 \pm 0.0077
\end{aligned}
$$

where the statistical and systematic errors of $\mathcal{A}_{\tau}$ and $\mathcal{A}_{\mathrm{e}}$ are added in quadrature before calculating the errors on $\hat{\mathrm{g}}_{v}^{\tau} / \hat{\mathrm{g}}_{a}^{\tau}$ and $\hat{\mathrm{g}}_{v}^{e} / \hat{\mathrm{g}}_{a}^{e}$. The agreement between these two values indicates that the data are consistent with the hypothesis of lepton universality. If universality is assumed, these results can be averaged to give:

$$
\sin ^{2} \theta_{\mathrm{eff}}^{\text {lept }}=0.2334 \pm 0.0012 .
$$

This measurement of $\sin ^{2} \theta_{\mathrm{eff}}^{\text {lept }}$ is of similar precision to other individual measurements at LEP using various techniques and is in agreement with the value of $\sin ^{2} \theta_{\text {eff }}^{\text {lept }}$ obtained from a Standard Model fit to all LEP electroweak data, including the $\tau$ polarization[31]. The measurement is more than two standard deviations higher than the value of $\sin ^{2} \theta_{\text {eff }}^{\text {lept }}$ from a measurement of $A_{L R}$ by the SLD collaboration[32], which gives the most precise single measurement of $\sin ^{2} \theta_{\text {eff }}^{\text {lept }}$ and which measures the same quantity as $\mathcal{A}_{\mathbf{e}}$. However, the $A_{L R}$ measurement is also more than two standard deviations lower than the Standard Model fit to all LEP electroweak data[31].

\section{Acknowledgements}

We particularly wish to thank the SL Division for the efficient operation of the LEP accelerator and for their continuing close cooperation with our experimental group. In addition to the support staff at our own institutions we are pleased to acknowledge the

Department of Energy, USA,

National Science Foundation, USA,

Particle Physics and Astronomy Research Council, UK,

Natural Sciences and Engineering Research Council, Canada,

Israel Ministry of Science,

Israel Science Foundation, administered by the Israel Academy of Science and Humanities,

Minerva Gesellschaft,

Japanese Ministry of Education, Science and Culture (the Monbusho) and a grant under the Monbusho International Science Research Program,

German Israeli Bi-national Science Foundation (GIF),

Direction des Sciences de la Matière du Commissariat à l'Energie Atomique, France,

Bundesministerium für Bildung, Wissenschaft, Forschung und Technologie, Germany,

National Research Council of Canada,

Hungarian Foundation for Scientific Research, OTKA T-016660, and OTKA F-015089. 


\section{References}

[1] S. Jadach and Z. Wass in Z Physics at LEP1, CERN 89-08, edited by G. Altarelli et al., Vol. 1 (1989) 235.

[2] OPAL Collab., K. Ahmet et al., Nucl. Inst. and Meth. A305 (1991) 275.

[3] OPAL Collab., R. Akers et al., Z. Phys. C65 (1995) 1.

[4] M. Davier et al., Phys. Lett. B306 (1993) 411.

[5] OPAL Collab., G. Alexander et al., Phys. Lett. B266 (1991) 201.

[6] S. Jadach, J.H. Kühn and Z. Wąs, Comp. Phys. Comm. 64 (1991) 275;

S. Jadach, B.F.L Ward and Z. Wa̧s, (KORALZ 3.8) Comp. Phys. Comm. 66 (1991) 276;

S. Jadach, B.F.L Ward and Z. Wa̧s, (KORALZ 4.0) Comp. Phys. Comm. 79 (1994) 503.

[7] M. Bohm, A. Denner and W. Hollik, Nucl. Phys. B304 (1988) 687;

F.A. Berends, R. Kleiss and W. Hollik, Nucl. Phys. B304 (1988) 712.

[8] T. Sjöstrand, Comp. Phys. Comm. 39 (1986) 347;

M. Bengtsson and T. Sjöstrand, Comp. Phys. Comm. 43 (1987) 367;

M. Bengtsson and T. Sjöstrand, Nucl. Phys. B289 (1987) 810.

[9] OPAL Collab., P. Acton et al., Z. Phys. C58 (1993) 387.

[10] R. Battacharya, J. Smith and G. Grammer, Phys. Rev. D15 (1977) 3267;

J. Smith, J.A.M. Vermaseren and G. Grammer, Phys. Rev. D15 (1977) 3280.

[11] J. Allison et al., Nucl. Inst. and Meth. A317 (1992) 47.

[12] R. Brun, F. Bruyant, M. Maire, A. C. McPherson, and P. Zanarini, GEANT3, CERN DD/EE/84-1 (1987).

[13] OPAL Collab., R. Akers et al., Phys. Lett. B328 (1994) 207.

For this analysis, the fine clustering selection II is used.

[14] M. Thomson, The Use of Maximum Entropy in Electromagnetic Calorimeter Event Reconstruction, CERN-PPE/96-055, submitted to Nucl. Inst. and Meth.

[15] ARGUS Collab., H. Albrecht et al., Z. Phys. C58 (1993) 61.

[16] J. H. Kühn, A. Santamaria Z. Phys. C48 (1990) 445;

J. H. Kühn and E. Mirkes, Z. Phys. C56 (1992) 661.

[17] R. Barlow and C. Beeston, Comp. Phys. Comm. 77 (1993) 219.

[18] OPAL Collab., M.Z. Akrawy et al., Phys. Lett. B240 (1990) 497;

OPAL Collab., G. Alexander et al., Z. Phys. C52 (1991) 175;

OPAL Collab., P.D. Acton et al., Z. Phys. C58 (1993) 219;

OPAL Collab., R. Akers et al., Z. Phys. C61 (1994) 19.

[19] D. Bardin, et al., CERN-TH. 6443/92 (1992). 
[20] Particle Data Group, Montanet et al., Phys. Rev. D50, (1994) 1173 and 1995 offyear partial update for the 1996 edition available on the PDG WWW pages (URL: http://pdg.lbl.gov/).

[21] ALEPH Collab., D. Buskulic et al., Z. Phys. C70 (1996) 579; ALEPH Collab., D. Buskulic et al., Z. Phys. C70 (1996) 561.

[22] DELPHI Collab., P. Abreu et al., Phys. Lett. B359 (1995) 411; DELPHI Collab., P. Abreu et al., Phys. Lett. B357 (1995) 715; DELPHI Collab., P. Abreu et al., Phys. Lett. B334 (1994) 435.

[23] L3 Collab., M. Acciarri et al., Phys. Lett. B352 (1995) 487;

L3 Collab., M. Acciarri et al., Phys. Lett. B345 (1995) 74.

[24] OPAL Collab., G. Alexander et al., Phys. Lett. B369 (1996) 163;

OPAL Collab., R. Akers et al., Z. Phys. C68 (1995) 555;

OPAL Collab., R. Akers et al., Z. Phys. C66 (1995) 543.

[25] OPAL Collab., R. Akers et al., Z. Phys. C67 (1995) 45.

[26] N. Isgur, C. Morningstar and C. Reader, Phys. Rev. D39 (1989) 1357.

[27] ALEPH Collab., D. Decamp et al., Phys. Lett. B265 (1991) 430;

ALEPH Collab., D. Buskulic et al., Z. Phys. C59 (1993) 369.

Using all published ALEPH $\tau$ polarization measurements, ALEPH quotes combined results of $\mathcal{A}_{\tau}=0.136 \pm 0.012 \pm 0.009, \mathcal{A}_{\mathbf{e}}=0.129 \pm 0.016 \pm 0.005, \sin ^{2} \theta_{\mathrm{eff}}^{\text {lept }}=0.2332 \pm 0.0014$.

[28] DELPHI Collab., P. Abreu et al., Z. Phys. C55 (1992) 555;

DELPHI Collab., P. Abreu et al., Z. Phys. C67 (1995) 183.

Using all published DELPHI $\tau$ polarization measurements, DELPHI quotes combined results of $\mathcal{A}_{\tau}=0.148 \pm 0.017 \pm 0.014, \mathcal{A}_{\mathbf{e}}=0.136 \pm 0.027 \pm 0.003, \sin ^{2} \theta_{\text {eff }}^{\text {lept }}=0.2320 \pm 0.0021$.

[29] L3 Collab., M. Acciarri et al., Phys. Lett. B341 (1994) 245;

L3 Collab., O. Adriani et al., Phys. Lett. B294 (1992) 466.

Using all published L3 $\tau$ polarization measurements, L3 quotes combined results of $\mathcal{A}_{\tau}=$ $0.150 \pm 0.013 \pm 0.009, \mathcal{A}_{\mathrm{e}}=0.157 \pm 0.020 \pm 0.005, \sin ^{2} \theta_{\mathrm{eff}}^{\mathrm{lept}}=0.2309 \pm 0.0016$.

[30] M. Consoli, W. Hollik and F. Jegerlehner in Z Physics at LEP1, CERN 89-08, edited by G. Altarelli et al., Vol. 1 (1989) 7.

[31] LEP Collaborations and the LEP Electroweak Working Group, A Combination of Preliminary LEP Electroweak Results and Constraints on the Standard Model, CERN-PPE/95172. The Standard Model fit to all LEP electroweak data, including the $\tau$ polarization, yields a value of $\sin ^{2} \theta_{\mathrm{eff}}^{\text {lept }}=0.23206 \pm 0.00028 \pm_{0.00017}^{0.00008}$. Some preliminary results are used as input to the fit, including a preliminary version of the measurement presented in this paper.

[32] SLD Collab., K. Abe et al, Phys. Rev. Lett. 73 (1994) 25. This measurement of $A_{L R}$ yields a value of $\sin ^{2} \theta_{\text {eff }}^{\text {lept }}=0.2292 \pm 0.0009 \pm 0.0004$.

The combination of this published $A_{L R}$ result with a recent SLD preliminary result obtained from the analysis of more data has been performed by SLD and is quoted in reference [31]. 
The value of $\sin ^{2} \theta_{\text {eff }}^{\text {lept }}$ obtained from $A_{\mathrm{LR}}$ and the value reported in this paper using $\tau$ polarization are in marginally better agreement when the preliminary $A_{L R}$ results are included. 


\section{OPAL}
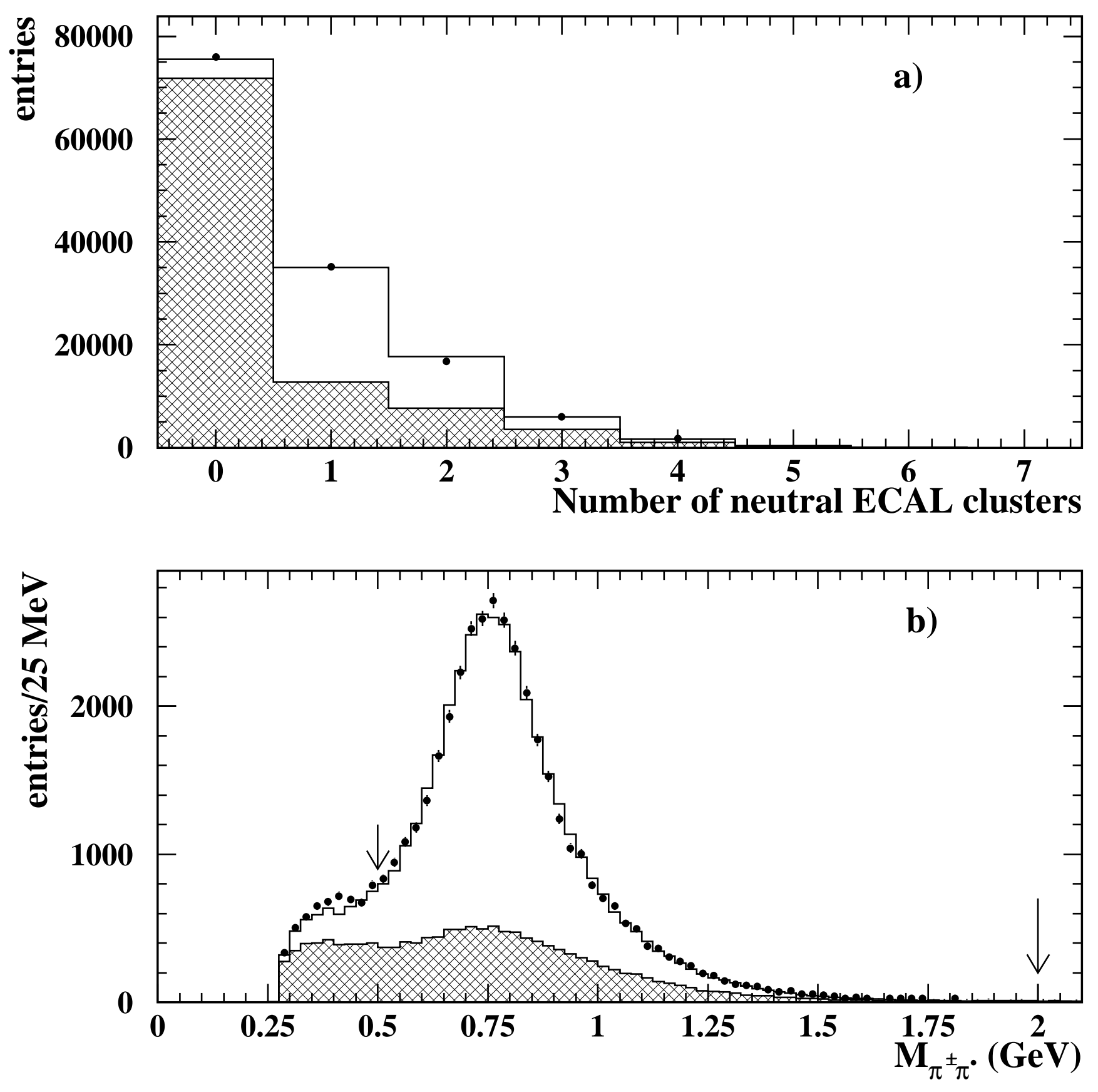

Figure 1: (a) The distribution of the number of ECAL clusters not associated with the track in one-prong $\tau$ decays. (b) Reconstructed mass of the $\tau \rightarrow \rho \nu_{\tau}$ candidates after applying all selection criteria other than that on the mass. In each figure the points with error bars represent the data, the open histogram the $\tau \rightarrow \rho \nu_{\tau}$ expectation from Monte Carlo and the hatched histogram the background contributions. The arrows indicate the range of masses required of the $\tau \rightarrow \rho \nu_{\tau}$ candidates in the analysis. 


\section{OPAL}
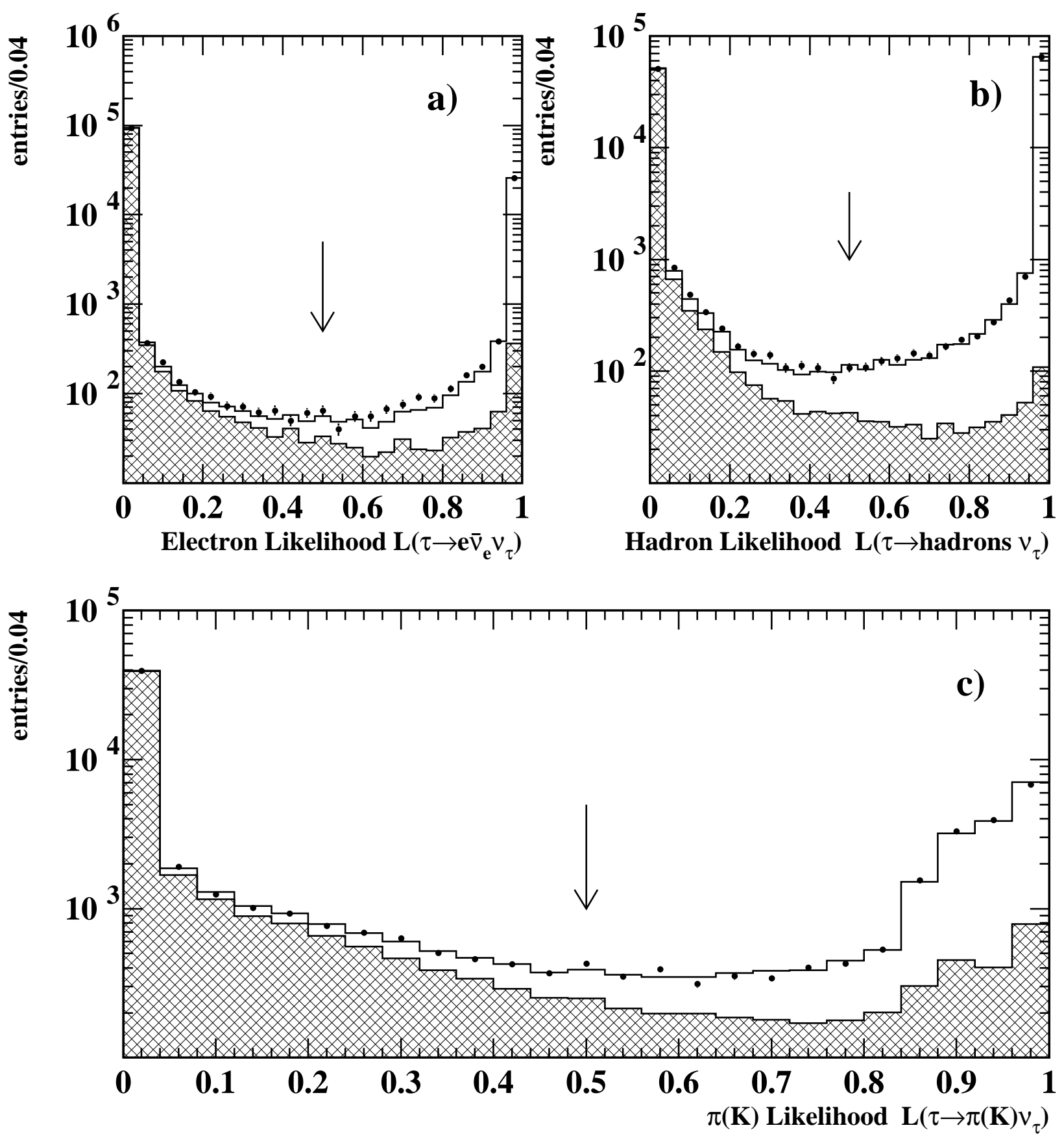

Figure 2: Distributions of relative likelihoods for (a) $\tau \rightarrow \mathrm{e} \bar{\nu}_{e} \nu_{\tau}$, (b) $\tau \rightarrow$ hadrons $\nu_{\tau}$, and (c) $\tau \rightarrow \pi(\mathrm{K}) \nu_{\tau}$. In each plot the points with error bars represent the data, the open histogram the expectation from Monte Carlo for the signal and the hatched histogram the expected contributions from non-signal sources. In each case selected decays are required to have $L>0.5$, as indicated by the arrow in each plot. 


\section{OPAL}
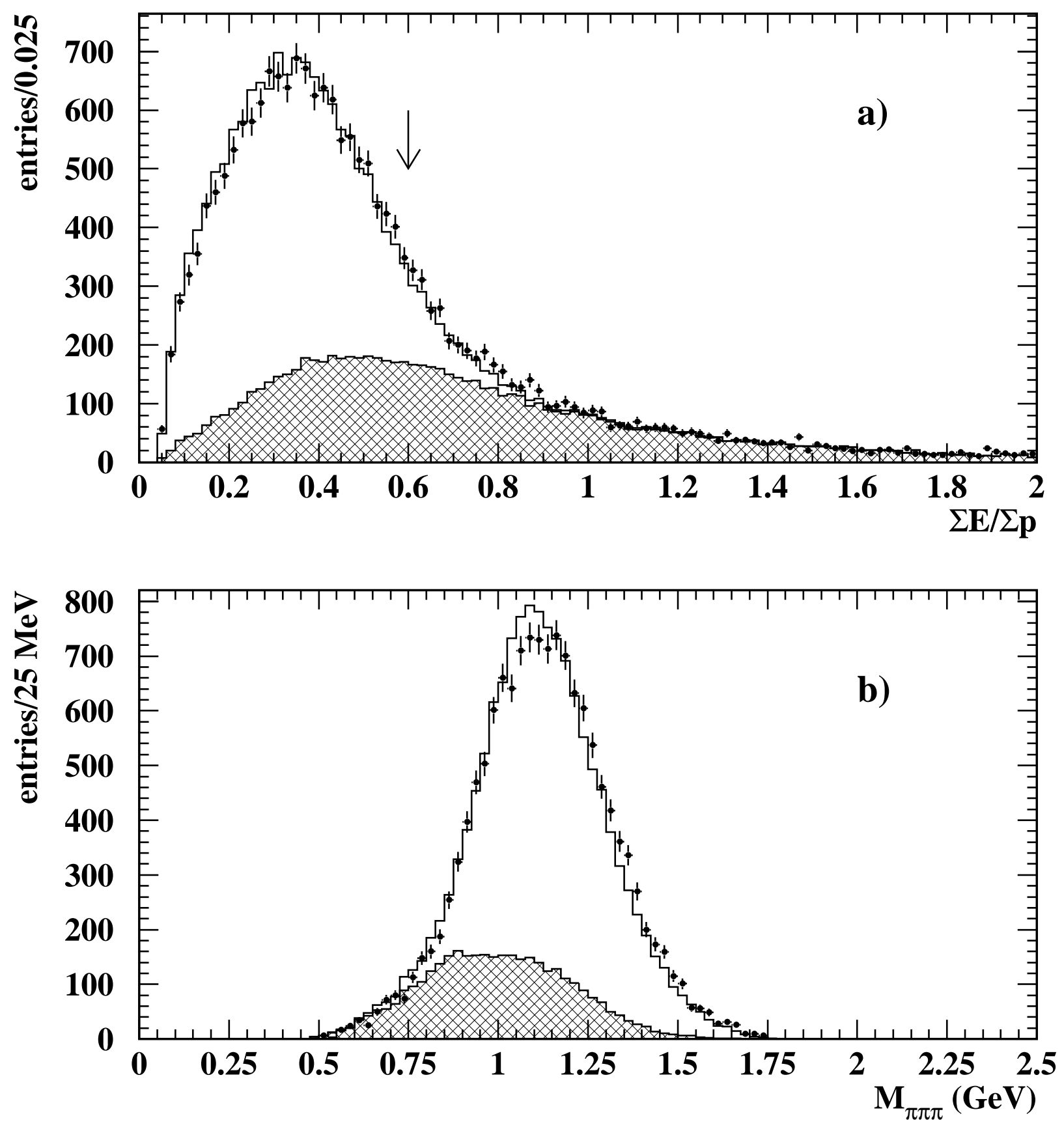

Figure 3: (a) The distribution of the total ECAL energy measured in the $\tau$-jet divided by the sum of the momenta of the three tracks present in the sample of three-prongs used in the $\tau \rightarrow \mathrm{a}_{1} \nu_{\tau}$ analysis after removing conversion electrons. The arrow indicates the position of the cut used to select the $\tau \rightarrow \mathbf{a}_{1} \nu_{\tau}$ sample. (b) Reconstructed mass of the three charged hadrons in the selected $\tau \rightarrow \mathrm{a}_{1} \nu_{\tau}$ sample under a pion hypothesis. The points with error bars represent the data, the open histogram the $\tau \rightarrow a_{1} \nu_{\tau}$ expectation from Monte Carlo and the hatched histogram the background contributions. 


\section{OPAL}
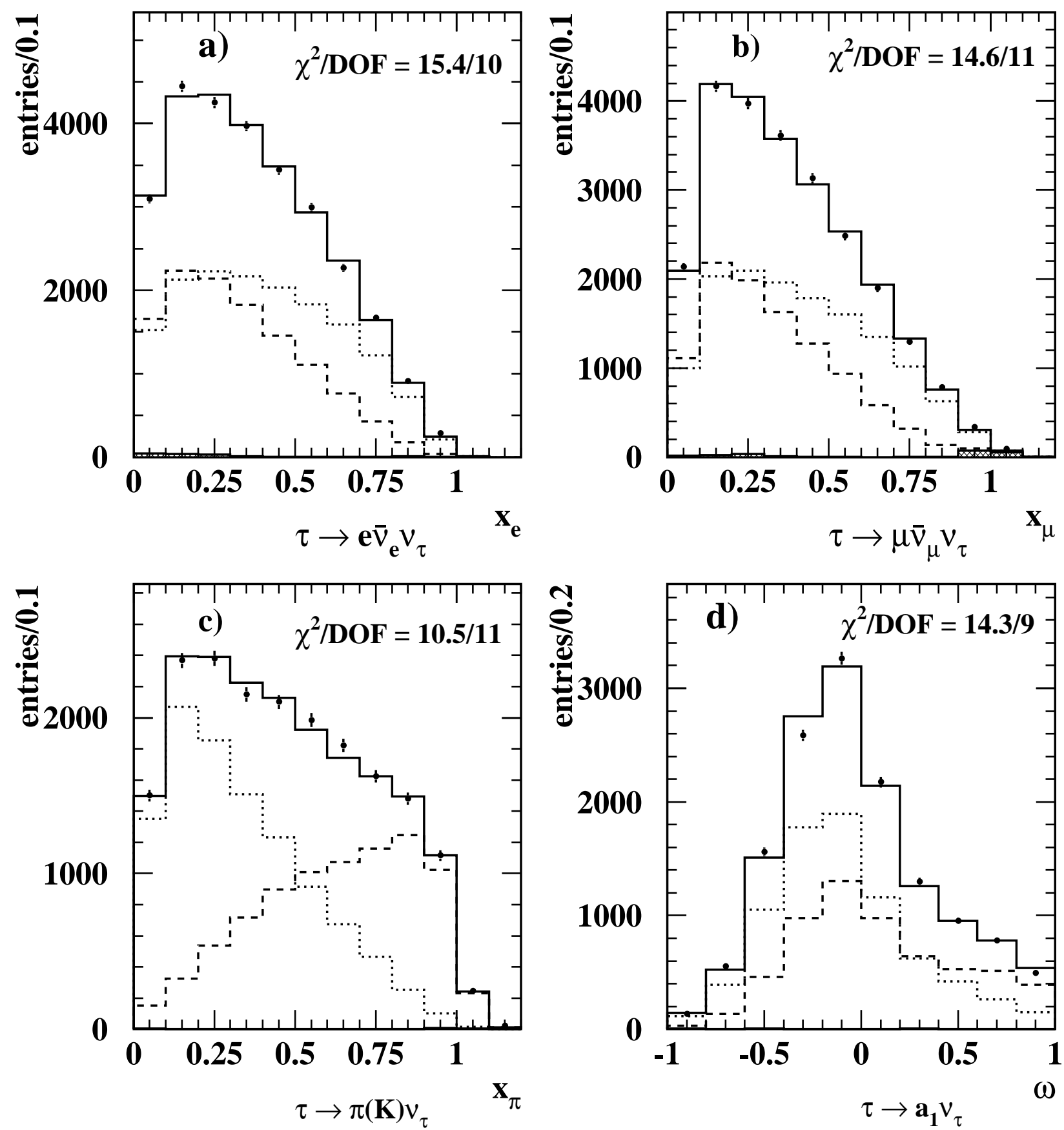

Figure 4: Distributions in the kinematic variables used in the fits as discussed in the text for the (a) $\tau \rightarrow \mathrm{e} \bar{\nu}_{e} \nu_{\tau}$, (b) $\tau \rightarrow \mu \bar{\nu}_{\mu} \nu_{\tau}$, (c) $\tau \rightarrow \pi(\mathrm{K}) \nu_{\tau}$ and (d) $\tau \rightarrow \mathrm{a}_{1} \nu_{\tau}$ channels where the data, shown by points with error bars, are integrated over the whole $\cos \theta_{\tau^{-}}$range. Overlaying these distributions are Monte Carlo distributions for the positive (dashed) and negative (dotted) helicity $\tau$ leptons and for their sum including non- $\tau$ background (solid), assuming a value of $\left\langle P_{\tau}\right\rangle=-13.0 \%$ as reported in the text. The small non- $\tau$ background is shown as a hatched histogram. The level of agreement between the data and Monte Carlo distributions is quantified by quoting the $\chi^{2}$ and the number of degrees of freedom. 


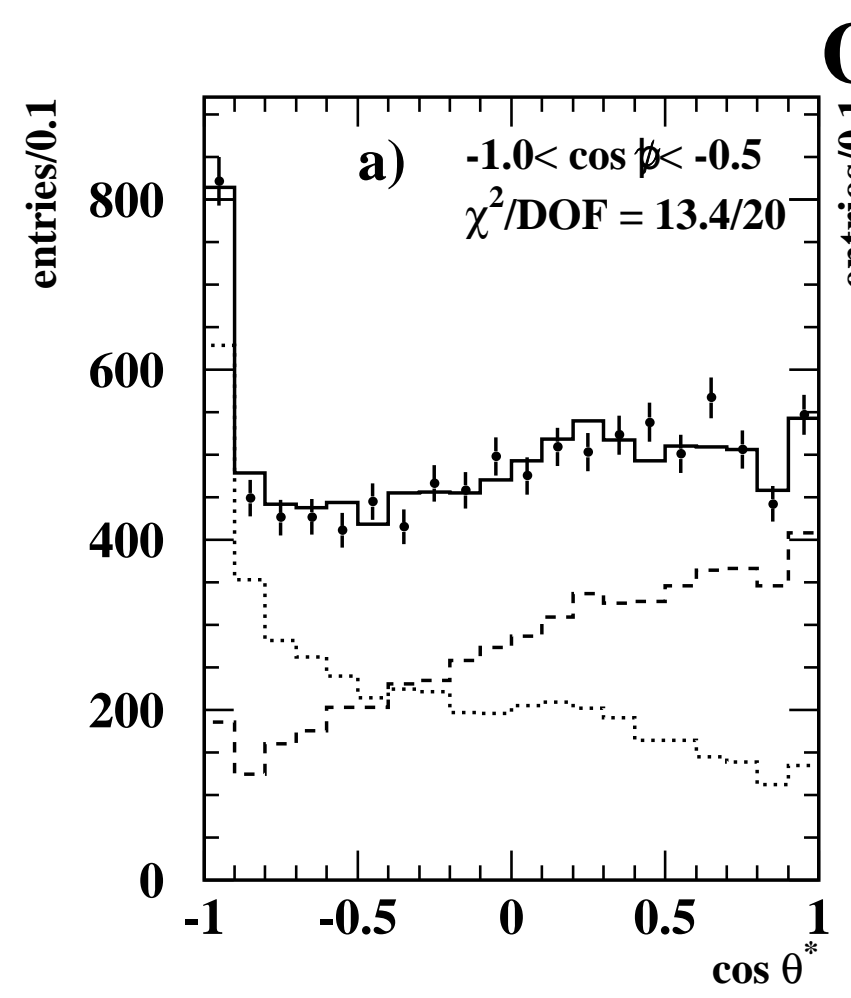

OPAL
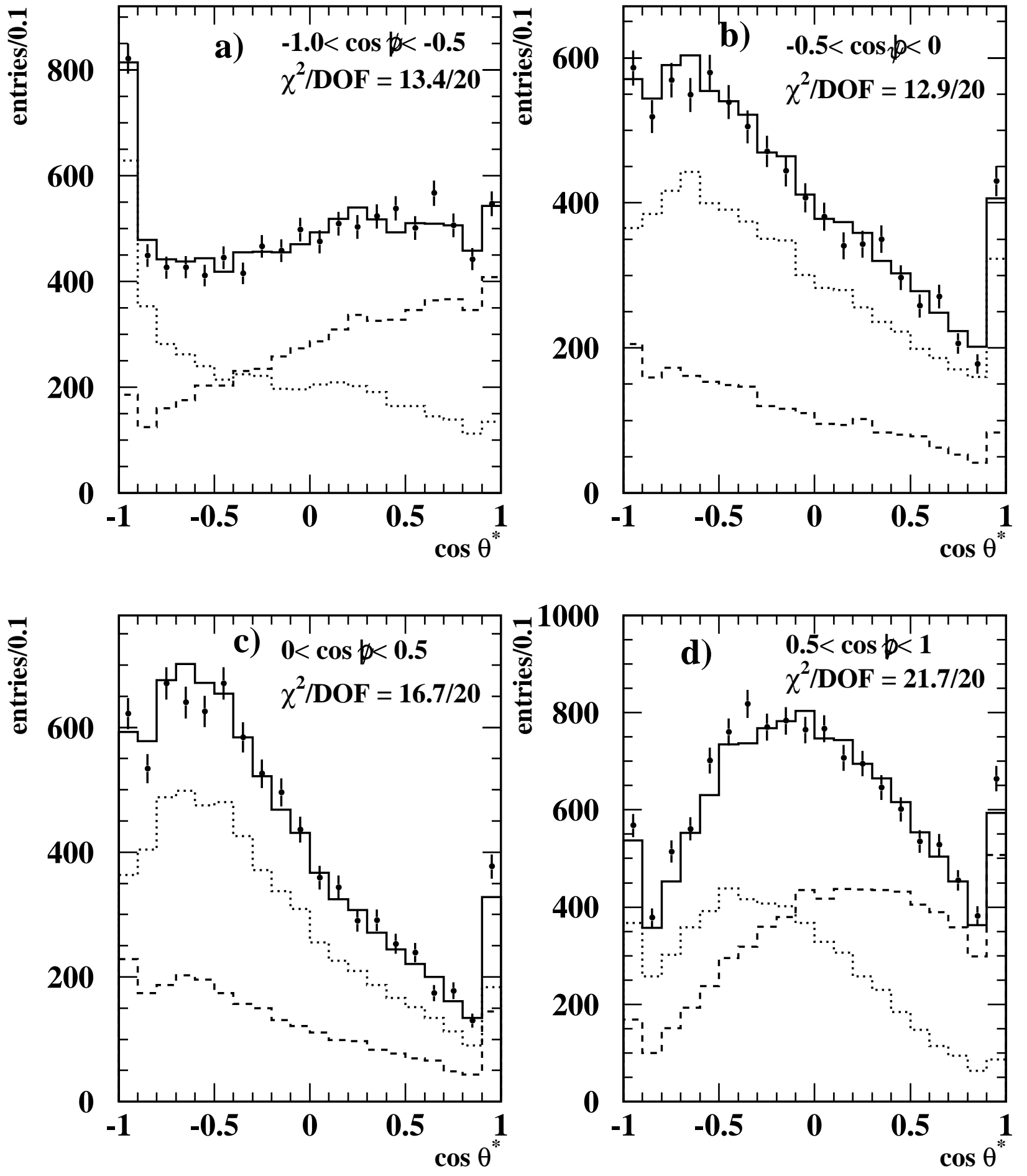

Figure 5: Distributions in $\cos \theta^{*}$ for four different bins in $\cos \psi$ for the $\tau \rightarrow \rho \nu_{\tau}$ analysis integrated over the whole $\cos \theta_{\tau^{-}}$range: (a) $-1.0<\cos \psi<-0.5$, (b) $-0.5<\cos \psi<0$, (c) $0<\cos \psi<0.5$, and (d) $0.5<$ $\cos \psi<1.0$. The points with error bars represent the data. The line histograms represent the Monte Carlo distributions for the positive (dashed) and negative (dotted) helicity $\tau$ leptons and for their sum (solid), assuming a value of $\left\langle P_{\tau}\right\rangle=-13.0 \%$ as reported in the text. The level of agreement between the data and Monte Carlo distributions is quantified by quoting the $\chi^{2}$ and the number of degrees of freedom. 


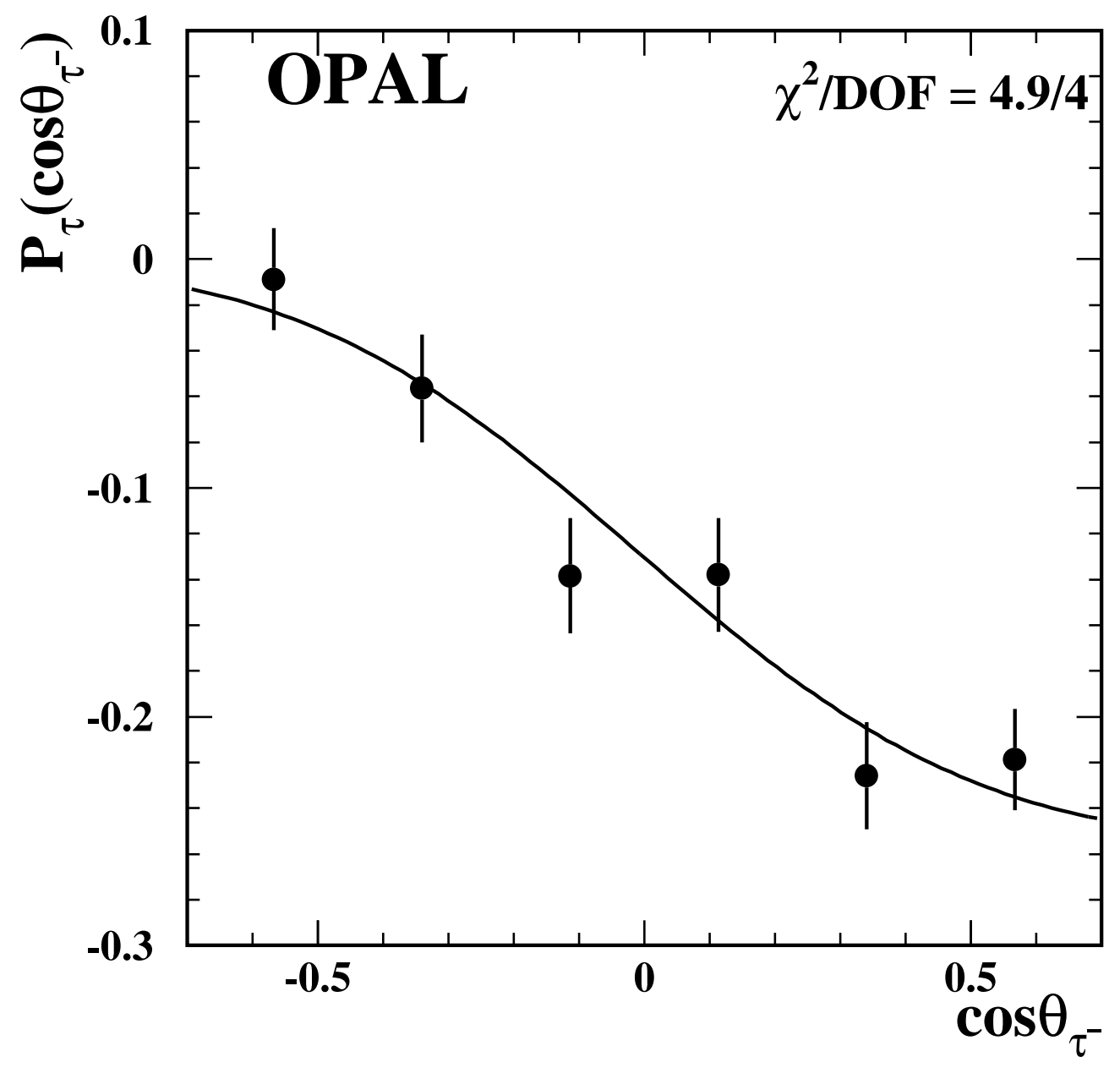

Figure 6: Tau polarization as a function of $\cos \theta_{\tau^{-}}$. The data points represent the $P_{\tau}$ values obtained from a global fit to all channels in each $\cos \theta_{\tau^{-}}$bin. The error bars represent data and Monte Carlo statistical errors only. The solid curve represents the expectation from the global fit result: $\left\langle P_{\tau}\right\rangle=$ $-13.0 \%$ and $\mathrm{A}_{\mathrm{pol}}^{\mathrm{FB}}=-9.4 \%$ as reported in the text. The level of agreement between the results of the fits in $\cos \theta_{\tau^{-}}$and the expectation from the global fit is quantified by quoting the $\chi^{2}$ and the number of degrees of freedom. 